\title{
Stabilized Multiscale Nonconforming Finite Element Method for the Stationary Navier-Stokes Equations
}

\author{
Tong Zhang, Shunwei $X u$, and Jien Deng \\ School of Mathematics and Information Science, Henan Polytechnic University, Jiaozuo 454003, China
}

Correspondence should be addressed to Tong Zhang, zhangtong0616@163.com

Received 22 May 2012; Accepted 8 August 2012

Academic Editor: Rudong Chen

Copyright (C) 2012 Tong Zhang et al. This is an open access article distributed under the Creative Commons Attribution License, which permits unrestricted use, distribution, and reproduction in any medium, provided the original work is properly cited.

We consider a stabilized multiscale nonconforming finite element method for the two-dimensional stationary incompressible Navier-Stokes problem. This method is based on the enrichment of the standard polynomial space for the velocity component with multiscale function and the nonconforming lowest equal-order finite element pair. Stability and existence uniqueness of the numerical solution are established, optimal-order error estimates are also presented. Finally, some numerical results are presented to validate the performance of the proposed method.

\section{Introduction}

As the development of science and technology, finite element method has became an important and powerful tool for the complex fluid problems, such as for the NavierStokes equations. It is well known that the pressure and velocity pairs satisfy the discrete Inf-Sup condition [1] that plays the key role for simulating the Navier-Stokes equations. However, some unstable mixed finite element pairs which violate the so-called Inf-Sup condition are also popular, see [2-4]. In order to overcome this restriction, various of stabilized methods have been proposed, including the bubble condensation-based methods [5], pressure projection method (PPM) [6-8], the local Gauss integration method (LGIM) [9-11], multiscale method [12, 13], macroelement stabilized method [3, 14], and so on. Most of these stabilized methods necessarily need to introduce the stabilization parameters either explicitly or implicitly. In addition, some of these techniques are conditionally stable or are of suboptimal accuracy. Therefore, the development of mixed finite element methods free from stabilization parameters has become increasingly important. 
In 2005, Franca et al. gave a new multiscale method for the reaction-diffusion equation in [15]. The chief characteristic of their method is to use the Petrov-Galerkin approach to split the solution into two parts, and the trial function space is enriched with an unstable bubble-like function, which is the solution to a local problem. Later, Barrenechea and Valentin [13] considered the relationship between the enriched multiscale method and stabilized techniques for generalized Stokes problem based on the $P_{1}-P_{1}$ pair. By enriching the velocity space with an unusual bubble function, Araya et al. established the convergence for the Stokes problem in [16], their method is different from usual residual free bubble method in [5], in which one should choose local basis functions to enrich the standard finite element spaces by solving some local problem analytically. Furthermore, the method proposed in [15] can also be used to treat the unsteady reaction-diffusion problem (see [17]).

Compared with conforming finite element method, the nonconforming finite element methods are more popular due to their simplicity and small support sets of basis functions. Crouzeix and Ravizrt in [18] used the nonconforming piecewise linear velocity and a piecewise constant pressure to solve the Stokes equations. In this paper, motivated by the ideas of $[13,15,16]$, we will use the Petrov-Galerkin approach based on the nonconforming velocity space to handle with the steady Navier-Stokes equations. The main differences between [13, $15,16,19]$ and this work lie in the following: (i) the finite element spaces of velocity are different; it is nonconforming element in this paper; (ii) the treated problems are different; we consider the nonlinear problem; (iii) the finite element pairs are different; the $\mathrm{NCP}_{1}-P_{1}$ pair is used in this paper.

The outline of this work is arranged as follows. In the following section, the abstract functional setting for steady Navier-Stokes equations is recalled. Section 3 is devoted to derive the general form of enriched multiscale method based on the $\mathrm{NCP}_{1}-P_{1}$ pair. After providing the stability and existence uniqueness for the approximation solution, the optimal error estimates are established in Section 4. In Section 5, Some numerical results are presented to verify the established theoretical analysis. Finally, Some conclusions are made in Section 6.

\section{Preliminaries}

Let $\Omega$ be an open bounded domain of $\mathbb{R}^{2}$ with Lipschitz continuous boundary $\partial \Omega$ and satisfy a further condition stated in (A1) below. The incompressible stationary Navier-Stokes equations with the homogeneous Dirichlet boundary condition are

$$
\begin{gathered}
-v \Delta u+(u \cdot \nabla) u+\nabla p=f \quad \text { in } \Omega, \\
\operatorname{div} u=0, \quad \text { in } \Omega \\
\left.u\right|_{\partial \Omega}=0 \quad \text { on } \partial \Omega
\end{gathered}
$$

where $u=\left(u_{1}(x), u_{2}(x)\right)^{T}$ represents the velocity, $p=p(x)$ the pressure, $f=f(x) \in L^{2}(\Omega)^{2}$ the prescribed body force, $v>0$ the viscosity coefficient.

In order to introduce the variational formulation for problem (2.1), we set

$$
\begin{gathered}
X=H_{0}^{1}(\Omega)^{2}, \quad Y=L^{2}(\Omega)^{2}, \quad D(A)=H^{2}(\Omega)^{2} \cap X, \\
M=L_{0}^{2}(\Omega)=\left\{q \in L^{2}(\Omega): \int_{\Omega} q d x=0\right\} .
\end{gathered}
$$


The standard notations of Sobolev space $W^{m, r}(\Omega)$ are used. To simplify, we use $H^{m}(\Omega)$ instead of $W^{m, r}(\Omega)$ as $r=2$ and $\|\cdot\|_{m}$ for $\|\cdot\|_{m, 2}$. The spaces $L^{2}(\Omega)^{m}(m=1,2)$ are endowed with the usual $L^{2}$-scalar product $(\cdot, \cdot)$ and $L^{2}$-norm $\|\cdot\|_{0}$. The spaces $H_{0}^{1}(\Omega)$ and $X$ are equipped with the scalar product $(\nabla u, \nabla v)$ and the norm $|u|_{1, \Omega}^{2}, u, v \in H_{0}^{1}(\Omega)$ (or $\left.X\right)$.

Define $A u=-\Delta u$ is the operator associated with the Navier-Stokes problem, it is positive self-adjoint operator from $D(A)$ onto $Y$.

Introducing the bilinear operator

$$
B(u, v)=(u \cdot \nabla) v+\frac{1}{2}(\operatorname{div} u) v \quad \forall u, v \in X
$$

and defining a trilinear form on $X \times X \times X$ as follows:

$$
\begin{aligned}
b(u, v, w)=\langle B(u, v), w\rangle_{X^{\prime} \times X} & =((u \cdot \nabla) v, w)+\frac{1}{2}((\operatorname{div} u) v, w) \\
& =\frac{1}{2}((u \cdot \nabla) v, w)-\frac{1}{2}((u \cdot \nabla) w, v) .
\end{aligned}
$$

The variational formulation of problem (2.1) reads as: find $(u, p) \in(X, M)$ such that for all $(v, q) \in(X, M)$

$$
a(u, v)-d(v, p)+d(u, q)+b(u, u, v)=(f, v)
$$

where

$$
\begin{gathered}
a(u, v)=v(\nabla u, \nabla v), \quad d(v, q)=-(v, \nabla q)=(q, \operatorname{div} v), \\
B_{0}((u, p) ;(v, q))=a(u, v)-d(v, p)+d(u, q)
\end{gathered}
$$

Clearly, the bilinear forms $a(\cdot, \cdot)$ and $d(\cdot, \cdot)$ are continuous on $X \times X$ and $X \times M$, respectively. Moreover, $d(\cdot, \cdot)$ also satisfies (see [20]):

$$
\sup _{0 \neq v \in X} \frac{|d(v, q)|}{|v|_{1, \Omega}} \geq \beta\|q\|_{0, \Omega^{\prime}}
$$

where $\beta$ is a positive constant depending only on $\Omega$.

It is easy to verify that $B_{0}$ satisfies the following important properties for all $(u, p)$, $(v, q) \in(X, M)($ see $[1])$ :

$$
\begin{gathered}
B_{0}((u, p) ;(u, p))=v\|u\|_{1, \Omega}^{2} \\
\left|B_{0}((u, p) ;(v, q))\right| \leq C\left(\|u\|_{1, \Omega}+\|p\|_{0, \Omega}\right)\left(\|v\|_{1, \Omega}+\|q\|_{0, \Omega}\right), \\
\beta_{0}\left(\|u\|_{1, \Omega}+\|p\|_{0, \Omega}\right) \leq \sup _{(v, q) \in(X, M)} \frac{\left|B_{0}((u, p) ;(v, q))\right|}{\|v\|_{1, \Omega}+\|q\|_{0, \Omega}}
\end{gathered}
$$


where $\beta_{0}>0$ is a constant. Here and below, the letter $C$ (with or without subscript) denotes a generic positive constant, depending at most on the data $v, \Omega$ and $f$. Furthermore, the following estimates about $b(\cdot, \cdot, \cdot)$ are hold $[1,20]$ :

$$
\begin{gathered}
b(u, v, w)=-b(u, w, v) \\
|b(u, v, w)| \leq \frac{1}{2} c_{0}\|u\|_{0, \Omega}{ }^{1 / 2}|u|_{1, \Omega}^{1 / 2}\left(|v|_{1, \Omega}|| w\left\|_{0, \Omega}^{1 / 2}|w|_{1, \Omega}^{1 / 2}+\right\| v \|_{0, \Omega}^{1 / 2}|v|_{1, \Omega}^{1 / 2}|w|_{1, \Omega}\right)
\end{gathered}
$$

for all $u, v, w \in X$ and

$$
|b(u, v, w)|+|b(v, u, w)|+|b(w, u, v)| \leq C|u|_{1, \Omega}\|A v\|_{0, \Omega}\|w\|_{0, \Omega}
$$

for all $u \in X, v \in D(A), w \in Y$.

As mentioned above, a further assumption about $\Omega$ is needed (see [1]).

(A1) Assume that $\Omega$ is regular so that the unique solution $(v, q) \in(X, M)$ of the steady Stokes equations

$$
-v \Delta v+\nabla q=g, \quad \operatorname{div} v=0 \text { in } \Omega,\left.\quad v\right|_{\partial \Omega}=0,
$$

for a prescribed $g \in Y$ exists and satisfies

$$
\|A v\|_{0, \Omega}+|q|_{1, \Omega} \leq C\|g\|_{0, \Omega}
$$

Under the assumption of (A1), if $\partial \Omega$ is of $C^{2}$ or $\Omega$ is a two-dimensional convex polygon, it has been shown that (see [20])

$$
\|v\|_{0, \Omega} \leq \gamma_{0}|v|_{1, \Omega}, \quad \forall v \in X, \quad|v|_{1, \Omega} \leq \gamma_{0}\|A v\|_{0, \Omega}, \quad \forall v \in D(A),
$$

where $\gamma_{0}$ is a positive constant only depending on $\Omega$.

The following existence and uniqueness results about problem (2.5) are classical (see $[1,20])$.

Theorem 2.1. Assume that $v$ and $f \in Y$ satisfy the following uniqueness condition:

$$
1-\frac{c_{0} r_{0}^{2}}{v^{2}}\|f\|_{0, \Omega}>0
$$

Then problem (2.5) admits a unique solution $(u, p) \in\left(D(A), H^{1}(\Omega) \cap M\right)$ with $\operatorname{div} u=0$ such that

$$
|u|_{1, \Omega} \leq \frac{\gamma_{0}}{v}\|f\|_{0, \Omega^{\prime}} \quad\|A u\|_{0, \Omega}+|p|_{1, \Omega} \leq C\|f\|_{0, \Omega}
$$




\section{Enriched Nonconforming Finite Element Method}

Let $\tau_{h}$ be a regular triangulation of $\Omega$ into element $\left\{K_{j}\right\}: \bar{\Omega}=\cup \bar{K}_{j}$, that is, $\left|K_{j}\right| \simeq C h_{K_{j}{ }^{\prime}}^{2}$ where $\left|K_{j}\right|$ is the area of the element $K_{j}$ and $h_{K_{j}}$ is the diameter of $K_{j}$; the mesh parameter $h$ is given by $h=\max \left\{h_{K_{j}}: K_{j} \in \tau_{h}\right\}$. Denote the boundary segment and the interior boundary by $\gamma_{j}=\partial \Omega \cap \partial K_{j}$ and $\gamma_{j k}=\gamma_{k j}=\partial K_{j} \cap \partial K_{k}$, respectively. Let $\Gamma_{h}$ and $\Gamma_{j}$ be the sets of $\gamma_{j k}$ and $\gamma_{j}$. The centers of $\gamma_{j}$ and $\gamma_{j k}$ are indicated by $\xi_{j}$ and $\xi_{j k}$, respectively. The finite element spaces investigated in this paper are the following mixed finite element spaces:

$$
\begin{gathered}
\mathrm{NCP}_{1}=\left\{v \in Y:\left.v\right|_{K_{j}} \in P_{1}\left(K_{j}\right)^{2}, v\left(\xi_{j k}\right)=v\left(\xi_{k j}\right), v\left(\xi_{j}\right)=0 \forall j, k, K_{j} \in \tau_{h}\right\}, \\
P_{1}=\left\{q \in H^{1}(\Omega):\left.q\right|_{K_{j}} \in P_{1}\left(K_{j}\right), \forall K_{j} \in \tau_{h}\right\},
\end{gathered}
$$

where $P_{1}\left(K_{j}\right)$ is the set of line polynomials on $K_{j}$, and noting that the nonconforming finite element space $\mathrm{NCP}_{1}$ is not a subspace of $X$. Defining the energy norm

$$
\|v\|_{1, h}=\left(\sum_{K_{j}}|v|_{1, K_{j}}^{2}\right)^{1 / 2}, \quad \forall v \in \mathrm{NCP}_{1}
$$

The finite element spaces $\mathrm{NCP}_{1}$ and $P_{1}$ satisfy the following approximation property (see $[4,21])$ : for $(v, q) \in H^{2}(\Omega) \times H^{1}(\Omega)$, there are two approximations $v_{I} \in \mathrm{NCP}_{1}$ and $q_{I} \in P_{1}$ such that

$$
\left\|v-v_{I}\right\|_{0, \Omega}+h\left(\left\|v-v_{I}\right\|_{1, h}+\left\|q-q_{I}\right\|_{0, \Omega}\right) \leq C h^{2}\left(\|A v\|_{0, \Omega}+|q|_{1, \Omega}\right),
$$

and the compatibility conditions hold for all $j$ and $k$ :

$$
\int_{\gamma_{j k}}[v] d s=0, \quad \int_{\Gamma_{j}} v d s=0 \quad \forall v \in \mathrm{NCP}_{1}
$$

where $[v]=v_{\gamma_{j k}}-v_{\gamma_{k j}}$ denotes the jump of the function $v$ across the boundary $\gamma_{j k}$.

Set $\langle\cdot, \cdot\rangle_{j}=(\cdot, \cdot)_{\partial K_{j}}$ and $|\cdot|_{m, j}=|\cdot|_{m, K_{j}}$. Then for all $u, v \in H^{1}\left(K_{j}\right)^{2}, q \in L^{2}(\Omega)$, the discrete bilinear forms are

$$
a_{h}(u, v)=\sum_{K_{j}} v(\nabla u, \nabla v)_{K_{j}} \quad d_{h}(v, p)=\sum_{K_{j}}(\operatorname{div} v, p)_{K_{j}} .
$$

For the nonconforming space $\mathrm{NCP}_{1}$, we define a local operator

$$
\Pi_{j}: H^{1}\left(K_{j}\right)^{2} \longrightarrow \mathrm{NCP}_{1}\left(K_{j}\right)
$$


satisfying

$$
\int_{\partial K_{j}}\left(v-\Pi_{j} v\right) d s=0
$$

Then the local operator $\Pi_{j}$ satisfies (see [21])

$$
\left|v-\Pi_{j} v\right|_{1, K_{j}} \leq C h^{i}|v|_{i+1, K_{j},} \quad v \in H^{i+1}\left(K_{j}\right), i=0,1, \quad\left\|\Pi_{j} v\right\|_{1, K_{j}} \leq C\|v\|_{1, K_{j}} .
$$

The global operator $\Pi_{h}: X \rightarrow \mathrm{NCP}_{1}$ is defined as $\left.\Pi_{h} v\right|_{j}=\Pi_{j} v, v \in X$.

As noted, the choice $\mathrm{NCP}_{1}-P_{1}$ is an unstable pair that does not satisfy the discrete Inf-Sup condition. Therefore, we need to introduce the enrichment multiscale method to overcome this restriction.

Let $E_{h}$ be a finite dimensional space, called multiscale space, such that

$$
E_{h} \in H^{1}\left(\tau_{h}\right)^{2}, \quad E_{h} \cap \mathrm{NCP}_{1}=\{0\}, \quad \text { where } H^{1}\left(\tau_{h}\right)^{2}=\left\{v \in Y:\left.v\right|_{K_{j}} \in H^{1}\left(K_{j}\right)^{2}\right\}
$$

The discrete weak formulation of the Stokes equations is to find $u_{h}+u_{e} \in \mathrm{NCP}_{1} \oplus E_{h}$ and $p_{h} \in P_{1}$, such that

$$
a_{h}\left(u_{h}+u_{e}, v_{h}\right)-d_{h}\left(v_{h}, p_{h}\right)+d_{h}\left(u_{h}+u_{e}, q_{h}\right)=(f, v)_{\Omega^{\prime}}
$$

for all $v_{h} \in \mathrm{NCP}_{1} \oplus H_{0}^{1}\left(\tau_{h}\right)^{2}$ and $q_{h} \in P_{1}$. Let $\left.u_{e}\right|_{K_{j}}=u_{e}^{K_{j}}+u_{e}^{\partial K_{j}}$, we can solve it through the following local problem:

$$
\begin{gathered}
-v \Delta u_{e}^{K_{j}}=f+v \Delta u_{h}-\nabla p_{h} \quad \text { in } K_{j},\left.\quad u_{e}^{K_{j}}\right|_{\partial K_{j}}=0, \\
-v \Delta u_{e}^{\partial K_{j}}=0 \quad \text { in } K_{j}, \quad u_{e}^{\partial K_{j}}=g_{e} \quad \text { on } \partial K_{j}, \\
-v \partial_{s s} g_{e}=\frac{1}{h_{e}}\left[v \partial_{n} u_{h}+p_{h} I \cdot n\right]_{E^{\prime}} \quad g_{e}=0 \text { at the nodes, }
\end{gathered}
$$

where $h_{e}$ denotes the length of the edge $E \in \partial K_{j} ; n$ the normal outward vector on $\partial K_{j} ; \partial_{s}, \partial_{n}$ are the tangential and normal derivative operators, respectively; $I$ is the $\mathbb{R}^{2 \times 2}$ identity matrix. Equation (3.11) is well posed, that is, $u_{e}$ can be expressed by $u_{h}, p_{h}$, and $f$ on each element $K_{j}$. For convenience, we define two local operators $\mathcal{M}_{K_{j}}: L^{2}\left(K_{j}\right)^{2} \rightarrow H_{0}^{1}\left(K_{j}\right)^{2}$ and $\mathscr{t}_{K_{j}}$ : $L^{2}\left(\partial K_{j}\right)^{2} \rightarrow H^{1}\left(K_{j}\right)^{2}$ by

$$
\begin{gathered}
u_{e}^{K_{j}}=\frac{1}{v} \mathcal{M}_{K_{j}}\left(f+v \Delta u_{h}-\nabla p_{h}\right), \quad \forall K_{j} \in \tau_{h}, \\
u_{e}^{\partial K}=\frac{1}{v} \mathscr{\ell}_{K_{j}}\left(\left[v \partial_{n} u_{h}+p_{h} I \cdot n\right]_{E}\right), \quad \forall K_{j} \in \tau_{h}, E \in \Gamma_{h} .
\end{gathered}
$$


With Green formulation and (3.12), for all $\left(u_{h}, p_{h}\right),\left(v_{h}, q_{h}\right) \in \mathrm{NCP}_{1} \times P_{1},(3.10)$ can be rewritten as

$$
\begin{aligned}
& \sum_{K_{j}}\left[v\left(\nabla u_{h}, \nabla v_{h}\right)_{K_{j}}-\left(p_{h}, \nabla \cdot v_{h}\right)_{K_{j}}+\left(q_{h}, \nabla \cdot u_{h}\right)_{K_{j}}\right] \\
& +\sum_{K_{j}} \frac{1}{v}\left(\mathcal{M}_{K_{j}}\left(-v \Delta u_{h}+\nabla p_{h}\right)-\mathscr{H}_{K_{j}}\left(\left[v \partial_{n} u_{h}+p_{h} I \cdot n\right]_{E}\right), v \Delta v_{h}+\nabla q_{h}\right)_{K_{j}} \\
& +\sum_{E \in \Gamma_{h}} \frac{1}{v}\left(\mathscr{H}_{K_{j}}\left(\left[v \partial_{h} u_{h}+p_{h} I \cdot n\right]_{E}\right), v \partial_{h} v_{h}+q_{h} I \cdot n\right)_{E} \\
& =\sum_{K_{j}}\left[\left(f, v_{h}\right)_{K_{j}}+\frac{1}{v}\left(\mathcal{M}_{K_{j}}(f), v \Delta v_{h}+\nabla q_{h}\right)_{K_{j}}\right] .
\end{aligned}
$$

With the help of (3.13), the enriched nonconforming finite element method for the stationary Navier-Stokes equations (2.1) is rewritten as follows: find $\left(u_{h}, p_{h}\right) \in \mathrm{NCP}_{1} \times P_{1}$ such that

$$
B\left(\left(u_{h}, p_{h}\right) ;\left(v_{h}, q_{h}\right)\right)+b\left(u_{h}, u_{h}, v_{h}\right)=F\left(v_{h}, q_{h}\right)
$$

for all $\left(v_{h}, q_{h}\right) \in \mathrm{NCP}_{1} \times P_{1}$, where

$$
\begin{aligned}
& B\left(\left(u_{h}, p_{h}\right) ;\left(v_{h}, q_{h}\right)\right)= B_{h}\left(\left(u_{h}, p_{h}\right) ;\left(v_{h}, q_{h}\right)\right)+\sum_{K_{j}} \frac{1}{v}\left(\mathcal{M}_{K_{j}}\left(\nabla p_{h}\right), \nabla q_{h}\right)_{K_{j}} \\
&+\sum_{E \in \Gamma_{h}} \frac{1}{v}\left(\mathcal{d}_{K_{j}}\left(\left[v \partial_{h} u_{h}\right]\right),\left[v \partial_{h} v_{h}\right]\right)_{E} \\
& \triangleq B_{h}\left(\left(u_{h}, p_{h}\right) ;\left(v_{h}, q_{h}\right)\right)_{\Omega}+\sum_{K_{j}} \tau_{K_{j}}\left(\nabla p_{h}, \nabla q_{h}\right)_{K_{j}} \\
&+\sum_{E \in \Gamma_{h}} \tau_{E}\left(\left[v \partial_{h} u_{h}\right],\left[v \partial_{h} v_{h}\right]\right)_{E}, \\
& F\left(v_{h}, q_{h}\right)= \sum_{K_{j}}\left[\left(f, v_{h}\right)_{K_{j}}+\frac{1}{v}\left(\mathcal{M}_{K_{j}}(f), \nabla q_{h}\right)_{K_{j}}\right], \\
& B_{h}\left(\left(u_{h}, p_{h}\right) ;\left(v_{h}, q_{h}\right)\right)=a_{h}\left(u_{h}, v_{h}\right)-d_{h}\left(v_{h}, p_{h}\right)+d_{h}\left(u_{h}, q_{h}\right) .
\end{aligned}
$$

By applying the technique to one used in [16], we can obtain that $\left(b_{K_{j}}, 1\right)_{K_{j}} /\left|K_{j}\right| \simeq$ $\widetilde{C} h_{K_{j}{ }^{\prime}}^{2}\left(a_{K_{j}}, 1\right)_{E} / h_{e} \bumpeq h_{e} / 12, \tau_{K_{j}} \bumpeq \widetilde{C} h_{K_{j}}^{2}$ and $\tau_{E} \backsim h_{e} /(12 v)$. Moreover, if $f$ is a piecewise constant, then we have $\mathcal{M}_{K_{j}}(f)=b_{K_{j}} f$,

$$
\left(\mathcal{M}_{K_{j}}(f), \nabla q_{h}\right)_{K_{j}}=\frac{\left(b_{K_{j}}, 1\right)_{K_{j}}}{\left|K_{j}\right|}\left(f, \nabla q_{h}\right)_{K_{j}} \simeq \widetilde{C} h_{K_{j}}^{2}\left(f, \nabla q_{h}\right)_{K_{j}} .
$$


Define the mesh-dependent norms as follows:

$$
\||u|\|_{h}^{2}=v\|u\|_{1, h}^{2}+\sum_{E \in \Gamma_{h}} \tau_{E}\left\|\left[v \partial_{n} u\right]\right\|_{0, E}^{2}, \quad\|q\|_{h}^{2}=\sum_{K_{j}} \tau_{K_{j}}|q|_{1, K_{j}}^{2}
$$

Remark 3.1. The assumption of piecewise constant $f$ is made simply to analyze the problem (3.14), but this assumption does not affect the precision of this method, and (3.14) may be implemented as it is presented for a general function $f \in L^{2}(\Omega)^{2}$. Here, we do not give the detail proof about this fact; readers can visit Appendix B of the paper [16] for $f \in H^{1}(\Omega)^{2}$.

Remark 3.2. Generally speaking, the following linear algebra equations can be obtained from the discrete system of original problem:

$$
\left(\begin{array}{cc}
A & -D \\
D^{T} & 0
\end{array}\right)\left(\begin{array}{l}
U \\
P
\end{array}\right)=\left(\begin{array}{l}
F \\
0
\end{array}\right)
$$

where the matrices $A$ and $D$ are deduced from the diffusion, convection, and incompressible terms; $F$ is the variation of the source term. The norm of matrix $A$ gets smaller as the convection increases; therefore, some unnecessary oscillations will be created. In order to eliminate these oscillations, we introduce the stabilized term, in this case, the coefficient matrix of discrete formulation transforms into

$$
\left(\begin{array}{cc}
A & -D \\
D^{T} & G
\end{array}\right)
$$

where $G$ is derived from the stabilized term, that is, the term of $\left(\nabla p_{h}, \nabla q_{h}\right)$. As the considered problem has strong convection, in order to obtain a good behavior of matrix $\left(\begin{array}{cc}A & -D \\ D^{T} & G\end{array}\right)$, we should choose a proper $G$. In this way, the singularly perturbed problem can be treated effectively. The reason that we treat the convection term not use enriched function technique is to simply the theoretical analysis and computation, and the discrete convection term has no influence about the stabilized term $G(\cdot, \cdot)$.

Lemma 3.3. Let $\left(v_{h}, q_{h}\right) \in \mathrm{NCP}_{1} \times \mathrm{P}_{1}$, then,

$$
B\left(\left(v_{h}, q_{h}\right) ;\left(v_{h}, q_{h}\right)\right)=\left\|\left|v_{h}\right|\right\|_{h}^{2}+\left\|q_{h}\right\|_{h}^{2} .
$$

Proof. The results follow from the definition of (3.15) and the mesh-dependent norms in each $K \in \tau_{h}$.

Before establishing the stability of scheme (3.14), we introduce the local trace theorem (see [1]). There exists $C>0$, independent of $h$, such that

$$
\|u\|_{0, \partial K_{j}}^{2} \leq C\left(h_{K_{j}}^{-1}\|u\|_{0, K_{j}}^{2}+h_{K_{j}}|u|_{1, K_{j}}^{2}\right), \quad \forall u \in H^{1}\left(K_{j}\right)
$$


Theorem 3.4. There exist two positive constants $C, \beta$ depending on $v$, for all $\left(u_{h}, p_{h}\right),\left(v_{h}, q_{h}\right) \in$ $\mathrm{NCP}_{1} \times \mathrm{P}_{1}$ such that

$$
\begin{gathered}
\left|B\left(\left(u_{h}, p_{h}\right) ;\left(v_{h}, q_{h}\right)\right)\right| \leq C\left(\left\|u_{h}\right\|_{1, h}+\left\|p_{h}\right\|_{0, \Omega}\right)\left(\left\|v_{h}\right\|_{1, h}+\left\|q_{h}\right\|_{0, \Omega}\right), \\
\sup _{0 \neq\left(v_{h}, q_{h}\right) \in\left(\mathrm{NCP}_{1}, P_{1}\right)} \frac{\left|B\left(\left(u_{h}, p_{h}\right) ;\left(v_{h}, q_{h}\right)\right)\right|}{\left\|v_{h}\right\|_{1, h}+\left\|q_{h}\right\|_{0, \Omega}} \geq \beta\left(\left\|u_{h}\right\|_{1, h}+\left\|p_{h}\right\|_{0, \Omega}\right) .
\end{gathered}
$$

Proof. It follows from $\left(u_{h}, p_{h}\right),\left(v_{h}, q_{h}\right) \in \mathrm{NCP}_{1} \times P_{1}$, inverse inequality, (3.15), and (3.21) that

$$
\begin{aligned}
\left|B\left(\left(u_{h}, p_{h}\right) ;\left(v_{h}, q_{h}\right)\right)\right| & \leq \\
\leq & v\left\|u_{h}\right\|_{1, h}\left\|v_{h}\right\|_{1, h}+\left\|u_{h}\right\|_{1, h}\left\|q_{h}\right\|_{0, \Omega}+\left\|p_{h}\right\|_{0, \Omega}\left\|v_{h}\right\|_{1, h} \\
& +\sum_{K_{j}} \tau_{K_{j}}\left|p_{h}\right|_{1, K_{j}}\left|q_{h}\right|_{1, K_{j}}+\sum_{E \in \Gamma_{h}} \tau_{E}\left\|\left[v \partial_{h} u_{h}\right]\right\|_{0, E}\left\|\left[v \partial_{h} v_{h}\right]\right\|_{0, E} \\
\leq & v\left\|u_{h}\right\|_{1, h}\left\|v_{h}\right\|_{1, h}+\left\|u_{h}\right\|_{1, h}\left\|q_{h}\right\|_{0, \Omega}+\left\|p_{h}\right\|_{0, \Omega}\left\|v_{h}\right\|_{1, h}+C_{1}\left\|p_{h}\right\|_{0, \Omega}\left\|q_{h}\right\|_{0, \Omega} \\
& +C_{2} \sum_{E \in \Gamma_{h}} \tau_{E}\left(h_{K_{j}}^{-1 / 2}\left\|\nabla u_{h}\right\|_{0, K_{j}}+h_{K_{j}}^{1 / 2}\left|\nabla u_{h}\right|_{1, K_{j}}\right)\left(h_{K_{j}}^{-1 / 2}\left\|\nabla v_{h}\right\|_{0, K_{j}}+h_{K_{j}}^{1 / 2}\left|\nabla v_{h}\right|_{1, K_{j}}\right) \\
\leq & C(v)\left\|u_{h}\right\|_{1, h}\left\|v_{h}\right\|_{1, h}+\left\|u_{h}\right\|_{1, h}\left\|q_{h}\right\|_{0, \Omega}+\left\|p_{h}\right\|_{0, \Omega}\left\|v_{h}\right\|_{1, h}+C_{1}\left\|p_{h}\right\|_{0, \Omega}\left\|q_{h}\right\|_{0, \Omega} \\
\leq & C\left(\left\|u_{h}\right\|_{1, h}+\left\|p_{h}\right\|_{0, \Omega}\right)\left(\left\|v_{h}\right\|_{1, h}+\left\|q_{h}\right\|_{0, \Omega}\right),
\end{aligned}
$$

that is, the continuity result (3.22) holds.

From the properties of the nonconforming finite element given in [18], for all $p_{h} \in$ $L^{2}\left(K_{j}\right)$, there exists a function $w \in H^{1}\left(K_{j}\right)^{2}$, such that $\|w\|_{1, h}=\left\|p_{h}\right\|_{0}$ and

$$
\left(\nabla \cdot w, p_{h}\right)_{K_{j}}=\left\|p_{h}\right\|_{0, K_{j}^{\prime}}^{2} \quad\|w\|_{1, h} \leq C\left\|p_{h}\right\|_{0, \Omega}
$$

Using the Cauchy-Schwartz inequality and (3.25), we have

$$
\begin{aligned}
\left|B\left(\left(u_{h}, p_{h}\right) ;(-w, 0)\right)\right| & =-v\left(\nabla u_{h}, \nabla w\right)+\left(p_{h}, \nabla \cdot w\right)-\sum_{E \in \Gamma_{h}} \tau_{E}\left(\left[v \partial_{h} u_{h}\right],\left[v \partial_{h} w\right]\right)_{E} \\
\geq & -v\left\|u_{h}\right\|_{1, h}\|w\|_{1, h}+C_{0}\|w\|_{1, h}\left\|p_{h}\right\|_{0, \Omega}-\sum_{E \in \Gamma_{h}} \tau_{E}\left\|\left[v \partial_{h} u_{h}\right]\right\|_{0, E}\left\|\left[v \partial_{h} w\right]\right\|_{0, E} \\
\geq & -\left(v\left\|u_{h}\right\|_{1, h}^{2}+\sum_{E \in \Gamma_{h}} \tau_{E}\left\|\left[v \partial_{h} u_{h}\right]_{0, E}^{2}\right\|\right)^{1 / 2}\left(v\|w\|_{1, h}^{2}+\sum_{E \in \Gamma_{h}} \tau_{E}\left\|\left[v \partial_{h} w\right]\right\|_{0, E}^{2}\right)^{1 / 2} \\
& +C_{0}\|w\|_{1, h}\left\|p_{h}\right\|_{0, h} .
\end{aligned}
$$


Using (3.21) and inverse inequality, we obtain that

$$
\begin{aligned}
\tau_{E}\left\|\left[v \partial_{n} w\right]\right\|_{0, E}^{2} & \leq \frac{h_{e}}{12 v}\left(h_{K}^{-1}\|v \nabla w \cdot n\|_{0, K}^{2}+h_{K}|v \nabla w \cdot n|_{1, K}^{2}\right) \\
& \leq \frac{h_{e} v}{12 h_{K}}|w|_{1, K}^{2}+\frac{C_{K} v h_{e}}{12 h_{K}}|w|_{1, K}^{2} \leq \frac{v\left(1+C_{K}\right)}{12}|w|_{1, K}^{2} .
\end{aligned}
$$

Combining (3.26) with (3.27) yields

$$
\begin{aligned}
& \left|B\left(\left(u_{h}, p_{h}\right) ;(-w, 0)\right)\right| \\
& \quad \geq-\sqrt{C v}\|w\|_{1, h}\left(v\left|u_{h}\right|_{1, h}^{2}+\sum_{E \in \Gamma_{h}} \tau_{E}\left\|\left[v \partial_{h} u_{h}\right]\right\|_{0, E}^{2}\right)^{1 / 2}+C_{0}\|w\|_{1, h}\left\|p_{h}\right\|_{0, \Omega} \\
& \quad=-\sqrt{C v}\|w\|_{1, h}\left\|\left|u_{h}\right|\right\|_{h}+C_{0}\left\|p_{h}\right\|_{0, \Omega}^{2} \\
& \quad \geq-C v r_{1}^{-1}\left\|\left|u_{h}\right|\right\|_{h}^{2}+\left(C_{0}-\gamma_{1}\right)\left\|p_{h}\right\|_{0, \Omega^{\prime}}^{2}
\end{aligned}
$$

where $C=\left(1+C_{0}\right) / 12$ with $C_{0}=\max _{k \in \tau_{h}} C_{K}$, and $\gamma_{1}$ is chosen small enough. Let

$$
\left(v_{h}, q_{h}\right)=\left(u_{h}-\delta w, p_{h}\right), \quad \delta>0 .
$$

Using (3.26) and Lemma 3.3 we have

$$
\begin{aligned}
& \left|B\left(\left(u_{h}, p_{h}\right) ;\left(v_{h}, q_{h}\right)\right)\right|=\left|B\left(\left(u_{h}, p_{h}\right) ;\left(u_{h}, p_{h}\right)\right)+\delta B\left(\left(u_{h}, p_{h}\right) ;(-w, 0)\right)\right| \\
& \quad \geq\left\|\left|u_{h}\right|\right\|_{h}^{2}+\left\|p_{h}\right\|_{h}^{2}+\delta\left(-C v \gamma_{1}^{-1}\left\|\left|u_{h}\right|\right\|_{h}^{2}+\left(C_{0}-\gamma_{1}\right)\left\|p_{h}\right\|_{0, \Omega}^{2}\right) \\
& \quad \geq\left(1-C \delta v \gamma_{1}^{-1}\right)\left\|u_{h} \mid\right\|_{h}^{2}+\left\|p_{h}\right\|_{h}^{2}+\delta\left(C_{0}-\gamma_{1}\right)\left\|p_{h}\right\|_{0, \Omega}^{2} \\
& \quad \geq v\left(1-C \delta v \gamma_{1}^{-1}\right)\left\|u_{h}\right\|_{1, h}^{2}+\delta\left(C_{0}-\gamma_{1}\right)\left\|p_{h}\right\|_{0, \Omega^{\prime}}^{2}
\end{aligned}
$$

provided that $0<\delta<\gamma_{1} /(C v)$ and $0<\gamma_{1}<C_{0}$. Denote

$$
C(v) \triangleq \min \left\{v\left(1-C \delta v \gamma_{1}^{-1}\right), \delta\left(C_{0}-\gamma_{1}\right)\right\}, \quad C(\delta) \triangleq \max \left\{2,1+2 \delta^{2}\right\}
$$

Then we have

$$
\begin{aligned}
\left\|v_{h}\right\|_{1, h}^{2}+\left\|q_{h}\right\|_{0, \Omega}^{2} & =\left\|u_{h}-\delta w\right\|_{1, h}^{2}+\left\|p_{h}\right\|_{0, \Omega}^{2} \\
& \leq 2\left\|u_{h}\right\|_{1, h}^{2}+\left(1+2 \delta^{2}\right)\left\|p_{h}\right\|_{0, \Omega}^{2} \\
& \leq C(\delta)\left(\left\|u_{h}\right\|_{1, h}^{2}+\left\|p_{h}\right\|_{0, \Omega}^{2}\right) .
\end{aligned}
$$

Taking $\beta=C(v) /(C(\delta))$, we obtain the desired result (3.23). 
Theorem 3.5. Under the assumptions of Theorem 2.1 and the following condition:

$$
\text { the strong uniqueness condition: } 1-c_{0} \gamma_{0} \frac{\gamma_{0}+v^{1 / 2}}{v^{2}}\|f\|_{0, \Omega}>0 \text {. }
$$

Problem (3.14) admits a unique solution $\left(u_{h}, p_{h}\right) \in\left(\mathrm{NCP}_{1}, \mathrm{P}_{1}\right)$, and satisfying

$$
\begin{gathered}
\left\|u_{h}\right\|_{1, h} \leq \frac{r_{0}+v^{1 / 2}}{v}\|f\|_{0, \Omega^{\prime}} \\
\left\|p_{h}\right\|_{0, \Omega} \leq \beta^{-1}\left[\left(r_{0}+\frac{C}{v}\right)\|f\|_{0, \Omega}+c_{0} \gamma_{0} v^{-2}\left(\gamma_{0}+v^{1 / 2}\right)^{2}\|f\|_{0, \Omega}^{2}\right] .
\end{gathered}
$$

Proof. Let Hilbert space $H_{h}=\left(\mathrm{NCP}_{1}, P_{1}\right)$ be with the scalar product and norm

$$
((v, q) ;(w, r))_{H_{h}}=\sum_{K_{j}}(\nabla v, \nabla w)_{K_{j}}+(q, r)
$$

and $K_{h}$ be a nonvoid, convex, and compact subset of $H_{h}$ defined by

$$
\begin{aligned}
K_{h}=\left\{(v, q) \in H_{h}:\|v\|_{1, h} \leq \frac{\gamma_{0}+v^{1 / 2}}{v}\|f\|_{0^{\prime}}\right. \\
\left.\quad\|q\|_{0, \Omega} \leq \frac{v \gamma_{0}+C}{\beta v}\|f\|_{0, \Omega}+\frac{c_{0} \gamma_{0}\left(\gamma_{0}+v^{1 / 2}\right)^{2}}{\beta v^{2}}\|f\|_{0, \Omega}^{2}\right\} .
\end{aligned}
$$

Defining a continuous mapping from $K_{h}$ into $H_{h}$ as follows: given $(\bar{v}, \bar{q}) \in K_{h}$, for all $(w, r) \in H_{h}$, find $(v, q)=F(\bar{v}, \bar{q})$ such that

$$
B((v, q) ;(w, r))+b(\bar{v}, v, w)=(f, w)+\sum_{K_{j}} \tau_{K_{j}}(f, \nabla r)_{K_{j}} .
$$

Taking $(w, r)=(v, q)$, using (2.8)-(2.13) and inverse inequality yields

$$
\begin{aligned}
v\|v\|_{1, h}^{2}+\|q\|_{h}^{2} & \leq\|v\|_{h}^{2}+\|q\|_{h}^{2} \leq \gamma_{0}\|f\|_{0, \Omega}\|v\|_{1, h}+\sum_{K_{j}} \tau_{K_{j}}\|f\|_{0, K_{j}}\|\nabla q\|_{0, K_{j}} \\
& \leq\left(\frac{r_{0}^{2}}{2 v}+\frac{h^{2}}{2}\right)\|f\|_{0, \Omega}^{2}+\frac{v}{2}\|v\|_{1, h}^{2}+\frac{1}{2} \sum_{K_{j}} \tau_{K_{j}}\|\nabla q\|_{0, K_{j}}^{2} \\
& =\left(\frac{r_{0}^{2}}{2 v}+\frac{h^{2}}{2}\right)\|f\|_{0, \Omega}^{2}+\frac{v}{2}\|v\|_{1, h}^{2}+\frac{1}{2}\|q\|_{h}^{2} .
\end{aligned}
$$


As a consequence, we have

$$
\|v\|_{1, h} \leq \frac{\gamma_{0}+v^{1 / 2}}{v}\|f\|_{0, \Omega}
$$

Using again (2.17), (3.23), (3.37), and inverse inequality, we arrive at

$$
\begin{aligned}
\beta\left(\|v\|_{1, h}+\|q\|_{0, \Omega}\right) & \leq \frac{|f, w|+\left|\sum_{K_{j}} \tau_{K_{j}}(f, \nabla r)_{K_{j}}\right|}{\|w\|_{1, h}+\|r\|_{0}}+c_{0} \gamma_{0}\|\bar{v}\|_{1, h}\|v\|_{1, h} \\
& \leq \gamma_{0}\|f\|_{0, \Omega}+\frac{C h}{v}\|f\|_{0, \Omega}+c_{0} \gamma_{0} v^{-2}\left(\gamma_{0}+v^{1 / 2}\right)^{2}\|f\|_{0, \Omega}^{2} \\
& \leq\left(\frac{\gamma_{0} v+C h}{v}\right)\|f\|_{0, \Omega}+c_{0} \gamma_{0} v^{-2}\left(\gamma_{0}+v^{1 / 2}\right)^{2}\|f\|_{0, \Omega}^{2} .
\end{aligned}
$$

Hence, the two estimates imply $(v, q)=F(\bar{v}, \bar{q}) \in K_{h}$, thanks to the fixed point theorem, the mapping $(v, q)=F(\bar{v}, \bar{q}) \in K_{h}$ has at least one fixed point $\left(u_{h}, p_{h}\right) \in K_{h} ;$ namely, $\left(u_{h}, p_{h}\right) \in K_{h}$ is a numerical solution of problem (3.14).

Next, we shall prove that the problem (3.14) has a unique solution $\left(u_{h}, p_{h}\right)$. In fact, if $\left(v_{h}, q_{h}\right)$ also satisfies $(3.14)$, then for all $(w, r) \in\left(\mathrm{NCP}_{1}, P_{1}\right)$ we have

$$
B\left(\left(u_{h}-v_{h}, p_{h}-q_{h}\right) ;(w, r)\right)=b\left(v_{h}-u_{h}, u_{h}, w\right)+b\left(v_{h}, v_{h}-u_{h}, w\right) .
$$

Taking $(w, r)=\left(u_{h}-v_{h}, p_{h}-q_{h}\right)$ in (3.41) and using again (2.8)-(2.13), Lemma 3.3, it follows that

$$
v\left\|u_{h}-v_{h}\right\|_{1, h}^{2} \leq c_{0} \gamma_{0}\left\|u_{h}\right\|_{1}\left\|u_{h}-v_{h}\right\|_{1, h}^{2} \leq c_{0} \gamma_{0} \frac{\gamma_{0}+v^{1 / 2}}{v}\|f\|_{0, \Omega}\left\|u_{h}-v_{h}\right\|_{1, h}^{2}
$$

Which, together with the strong uniqueness condition

$$
v-c_{0} \gamma_{0} \frac{\gamma_{0}+v^{1 / 2}}{v}\|f\|_{0, \Omega}=v\left(1-c_{0} \gamma_{0} \frac{\gamma_{0}+v^{1 / 2}}{v^{2}}\|f\|_{0, \Omega}\right)>0
$$

gives $u_{h}=v_{h}$. Using again (2.13), (3.23), and (3.41), we obtain $\beta\left\|p_{h}-q_{h}\right\|_{0, \Omega}^{2} \leq 0$ which implies $p_{h}=q_{h}$.

\section{Error Estimates}

In order to derive the error estimates of the numerical solution $\left(u_{h}, p_{h}\right)$, we introduce the Galerkin projection $\left(R_{h}, Q_{h}\right):(X, M) \rightarrow\left(\mathrm{NCP}_{1}, P_{1}\right)$ defined as follows: for all $\left(v_{h}, q_{h}\right) \in$ $\left(\mathrm{NCP}_{1}, P_{1}\right)$

$$
B\left(\left(R_{h}(v, q), Q_{h}(v, q)\right) ;\left(v_{h}, q_{h}\right)\right)=B_{0}\left((v, q) ;\left(v_{h}, q_{h}\right)\right) .
$$

Noting the Theorem 3.4, $\left(R_{h}(v, q), Q_{h}(v, q)\right)$ is well defined.

By using a similar argument to the one used in [14, 22], we have the following lemma. 
Lemma 4.1. Let $(u, p) \in D(A) \times\left(H^{1}(\Omega) \cap M\right)$; under the assumptions of Theorems 3.4 and 3.5 , the projection operator $\left(R_{h}, Q_{h}\right)$ satisfies

$$
\left\|u-R_{h}(u, p)\right\|_{0, \Omega}+h\left(\left\|u-R_{h}(u, p)\right\|_{1, h}+\left\|p-Q_{h}(u, p)\right\|_{0, \Omega}\right) \leq C h^{2}\left(\|A u\|_{0, \Omega}+|p|_{1, \Omega}\right) .
$$

Proof. From $(u, p) \in\left[H^{2}(\Omega)^{2} \cap X\right] \times\left[H^{1}(\Omega) \cap M\right]$, we have $\left[v \partial_{n} u\right]_{E}=0$. For all $\left(v_{h}, q_{h}\right) \in$ $\mathrm{NCP}_{1} \times P_{1}$, using (4.1) yields

$$
\begin{aligned}
B\left(\left(R_{h}(u, p), Q_{h}(u, p)\right)\left(v_{h}, q_{h}\right)\right) & =B_{0}\left((u, p) ;\left(v_{h}, q_{h}\right)\right) \\
& =B\left((u, p) ;\left(v_{h}, q_{h}\right)\right)-\sum_{K_{j}} \tau_{K_{j}}\left(\nabla p, \nabla q_{h}\right)_{K_{j}} .
\end{aligned}
$$

From the definition of $\left(R_{h}(u, p), Q_{h}(u, p)\right),(3.3)$, combining Theorem 3.4, (4.3), the triangular with inverse inequalities, we arrive at

$$
\begin{aligned}
& \left\|u-R_{h}(u, p)\right\|_{1, h}+\left\|p-Q_{h}(u, p)\right\|_{0, \Omega} \\
& \leq\left\|u-u_{I}\right\|_{1, h}+\left\|p-p_{I}\right\|_{0, \Omega}+\left\|u_{I}-R_{h}(u, p)\right\|_{1, h}+\left\|p_{I}-Q_{h}(u, p)\right\|_{0, \Omega} \\
& \leq\left\|u-u_{I}\right\|_{1, h}+\left\|p-p_{I}\right\|_{0, \Omega}+\beta^{-1} \sup _{\left(v_{h}, q_{h}\right) \in N C P_{1} \times P_{1}} \frac{\left|B\left(\left(u_{I}-R_{h}(u, p), p_{I}-Q_{h}(u, p)\right) ;\left(v_{h}, q_{h}\right)\right)\right|}{\left\|v_{h}\right\|_{1, h}+\left\|q_{h}\right\|_{0, \Omega}} \\
& \leq \beta^{-1} \sup _{\left(v_{h}, q_{h}\right) \in \mathrm{NCP} P_{1} \times P_{1}} \frac{\left|B\left(\left(u_{I}-u, p_{I}-p\right) ;\left(v_{h}, q_{h}\right)\right)\right|+\left|\sum_{K_{j}} \tau_{K_{j}}\left(\nabla p, \nabla q_{h}\right)_{K_{j}}\right|}{\left\|v_{h}\right\|_{1, h}+\left\|q_{h}\right\|_{0, \Omega}} \\
& \quad+\left\|u-u_{I}\right\|_{1, h}+\left\|p-p_{I}\right\|_{0, \Omega} .
\end{aligned}
$$

It is easy to check that

$$
\left|B\left(\left(u_{I}-u, p_{I}-p\right) ;\left(v_{h}, q_{h}\right)\right)\right| \leq C\left(\left\|u-u_{I}\right\|_{1, h}+\left\|p-p_{I}\right\|_{0, \Omega}+h|p|_{1}\right)\left(\left\|v_{h}\right\|_{1, h}+\left\|q_{h}\right\|_{0, \Omega}\right) .
$$


Combining (4.4), (4.5), and inverse inequality yields

$$
\begin{aligned}
\| u- & R_{h}(u, p)\left\|_{1, h}+\right\| p-Q_{h}(u, p) \|_{0, \Omega} \\
& \leq C\left(\left\|u-u_{I}\right\|_{1, h}+\left\|p-p_{I}\right\|_{0, \Omega}+h|p|_{1}\right)+\beta^{-1} \sup _{\left(v_{h}, q_{h}\right) \in \mathrm{NCP} P_{1} \times P_{1}} \frac{\left|\sum_{K_{j}} \tau_{K_{j}}\left(\nabla p, \nabla q_{h}\right)_{K_{j}}\right|}{\left\|v_{h}\right\|_{1, h}+\left\|q_{h}\right\|_{0, \Omega}} \\
& \leq C_{1} h\left(\|A u\|_{0, \Omega}+|p|_{1, \Omega}\right)+C_{2} h \sup _{\left(v_{h}, q_{h}\right) \in \mathrm{NCP} P_{1} \times P_{1}} \frac{\sum_{K_{j}} h_{K_{j}}\|\nabla p\|_{0, K_{j}}\left\|\nabla q_{h}\right\|_{0, K_{j}}}{\left\|v_{h}\right\|_{1, h}+\left\|q_{h}\right\|_{0, \Omega}} \\
& \leq C h\left(\|A u\|_{0, \Omega}+\|p\|_{1, \Omega}\right) .
\end{aligned}
$$

In order to derive the estimate in the $L^{2}$-norm, we consider the following dual problem with $(e, \eta)=\left(u-R_{h}(u, p), p-Q_{h}(u, p)\right):$

$$
\begin{gathered}
-\Delta \Phi+\nabla \Psi=e \quad \text { in } \Omega \\
\operatorname{div} \Phi=0 \quad \text { in } \Omega, \\
\left.\Phi\right|_{\partial \Omega}=0 \quad \text { on } \partial \Omega .
\end{gathered}
$$

Based on the assumption of (A1), (4.7)-(4.9) have a unique solution and satisfy

$$
\|A \Phi\|_{0, \Omega}+|\Psi|_{1, \Omega} \leq C\left\|u-R_{h}(u, p)\right\|_{0, \Omega} .
$$

Multiplying (4.7) and (4.8) by $e$ and $\eta$, respectively, integrating over $\Omega$, and using (4.3) with $\left(v_{h}, q_{h}\right)=\left(\Phi_{I}, \Psi_{I}\right)$, we see that

$$
\begin{aligned}
\| u- & R_{h}(u, p) \|_{0}^{2} \\
= & B_{0}\left(\left(u-R_{h}(u, p), p-Q_{h}(u, p)\right) ;(\Phi, \Psi)\right) \\
& -\sum_{K_{j}}\left\langle\frac{\partial \Phi}{\partial n}, u-R_{h}(u, p)\right\rangle_{j}+\sum_{K_{j}}\left\langle\left(u-R_{h}(u, p)\right) \cdot n, \Psi\right\rangle_{j} \\
= & B\left(\left(u-R_{h}(u, p), p-Q_{h}(u, p)\right) ;(\Phi, \Psi)\right)-\sum_{K_{j}} \tau_{K_{j}}\left(\nabla\left(p-Q_{h}(u, p)\right), \nabla \Psi\right)_{K_{j}} \\
& -\sum_{K_{j}}\left\langle\frac{\partial \Phi}{\partial n}, u-R_{h}(u, p)\right\rangle_{j}+\sum_{K_{j}}\left\langle\left(u-R_{h}(u, p)\right) \cdot n, \Psi\right\rangle_{j}
\end{aligned}
$$


Abstract and Applied Analysis

$$
\begin{aligned}
= & B\left(\left(u-R_{h}(u, p), p-Q_{h}(u, p)\right) ;\left(\Phi-\Phi_{I}, \Psi-\Psi_{I}\right)\right) \\
& -\sum_{K_{j}} \tau_{K_{j}}\left(\nabla\left(p-Q_{h}(u, p)\right), \nabla \Psi\right)_{K_{j}}+\sum_{K_{j}} \tau_{K_{j}}\left(\nabla p, \nabla \Psi_{I}\right)_{K_{j}} \\
& -\sum_{K_{j}}\left\langle\frac{\partial \Phi}{\partial n}, u-R_{h}(u, p)\right\rangle_{j}+\sum_{K_{j}}\left\langle\left(u-R_{h}(u, p)\right) \cdot n, \Psi\right\rangle_{j},
\end{aligned}
$$

where $\left(\Phi_{I}, \Psi_{I}\right)$ is the finite element interpolation of $(\Phi, \Psi)$ in $\left(\mathrm{NCP}_{1}, P_{1}\right)$ and satisfies (3.3). For each $E \in \partial K_{j}$, we define the mean value of $u-R_{h}(u, p)$ and $\Psi$ on $E$

$$
\overline{u-R_{h}(u, p)}=\left.\frac{1}{h_{e}} \int_{E}\left(u-R_{h}(u, p)\right)\right|_{K_{j}} d s ; \quad \bar{\Psi}=\left.\frac{1}{h_{e}} \int_{E} \Psi\right|_{K_{j}} d s .
$$

Note that each interior edge appears twice in the sum of $(4.11) ; \overline{u-R_{h}(u, p)}$ and $\bar{\Psi}$ are constants. Then it follows from (4.11) that

$$
\begin{aligned}
\| u- & R_{h}(u, p) \|_{0, \Omega}^{2} \\
= & B\left(\left(u-R_{h}(u, p), p-Q_{h}(u, p)\right) ;\left(\Phi-\Phi_{I}, \Psi-\Psi_{I}\right)\right)+\sum_{K_{j}} \tau_{K_{j}}\left(\nabla p, \nabla \Psi_{I}\right)_{K_{j}} \\
& -\sum_{K_{j}} \tau_{K_{j}}\left(\nabla\left(p-Q_{h}(u, p)\right), \nabla \Psi\right)_{K_{j}}+\sum_{K_{j}} \sum_{E \in \partial K_{j}}\left(\left(u-R_{h}(u, p)\right) \cdot n, \Psi-\bar{\Psi}\right)_{E} \\
& -\sum_{K_{j}} \sum_{E \in \partial K_{j}}\left(\frac{\partial \Phi}{\partial n}, u-R_{h}(u, p)-\overline{u-R_{h}(u, p)}\right)_{E}
\end{aligned}
$$

Combining (3.3) with Lemma 4.1, we deduce that

$$
\begin{aligned}
& B\left(\left(u-R_{h}(u, p), p-Q_{h}(u, p)\right) ;\left(\Phi-\Phi_{I}, \Psi-\Psi_{I}\right)\right) \\
& \leq C h^{2}\left(\|A u\|_{0_{1, \Omega}}+|p|_{1, \Omega}\right)\left(\|A \Phi\|_{0, \Omega}+|\Psi|_{1, \Omega}\right) \\
& \sum_{K_{j}} \tau_{K_{j}}\left(\nabla p, \nabla \Psi_{I}\right)_{K_{j}}-\sum_{K_{j}} \tau_{K_{j}}\left(\nabla\left(p-Q_{h}(u, p)\right), \nabla \Psi\right)_{K_{j}} \\
& \quad \leq \sum_{K_{j}} \tau_{K_{j}}|p|_{1, K_{j}}\left|\Psi_{I}\right|_{1, K_{j}}+\sum_{K_{j}} \tau_{K_{j}}\left|p-Q_{h}(u, p)\right|_{1, K_{j}}|\Psi|_{1, K_{j}} .
\end{aligned}
$$

With the help of (4.12), we have

$$
\int_{E}\left[\left(u-R_{h}(u, p)\right)-\overline{u-R_{h}(u, p)}\right] d s=0 .
$$


Combining the definition of $\Pi_{j}$, (4.16), and local trace theorem (3.21) with the standard argument for the nonconforming element (see [21]), we see that

$$
\begin{aligned}
& \sum_{K_{j}} \sum_{E \in \partial K_{j}}\left(\frac{\partial \Phi}{\partial n}, u-R_{h}(u, p)-\overline{u-R_{h}(u, p)}\right)_{E} \\
& \quad=\sum_{K_{j}} \sum_{E \in \partial K_{j}}\left(\frac{\partial \Phi}{\partial n}-\frac{\partial\left(\Pi_{j} \Phi\right)}{\partial n}, u-R_{h}(u, p)-\overline{u-R_{h}(u, p)}\right)_{E} \\
& \quad \leq \sum_{K_{j}} \sum_{E \in \partial K_{j}}\left\|\nabla\left(\Phi-\Pi_{j} \Phi\right)\right\|_{L^{2}(E)}\left\|u-R_{h}(u, p)-\overline{u-R_{h}(u, p)}\right\|_{L^{2}(E)} \\
& \quad \leq C h^{2}\left(\|A u\|_{0, \Omega}+|p|_{1, \Omega}\right)\|A \Phi\|_{0, \Omega} .
\end{aligned}
$$

In a similar way, we have

$$
\sum_{K_{j}} \sum_{E \in \partial K_{j}}\left(\left(u-R_{h}(u, p)\right) \cdot n, \Psi-\bar{\Psi}\right)_{E} \leq C h^{2}\left(\|A u\|_{0, \Omega}+|p|_{1}\right)|\Psi|_{1, \Omega}
$$

By combining (4.13)-(4.15) with (4.17)-(4.18), we deduce that

$$
\left\|u-R_{h}(u, p)\right\|_{0, \Omega} \leq C h^{2}\left(\|A u\|_{0, \Omega}+|p|_{1, \Omega}\right),
$$

which, together with (4.6). We finish the proof.

Theorem 4.2. Assume that the conditions of Theorems 3.4 and 3.5 are valid; let $(u, p),\left(u_{h}, p_{h}\right)$ be the solutions of (2.1) and (3.14), respectively, then

$$
\left\|u-u_{h}\right\|_{1, h}+\left\|p-p_{h}\right\|_{0, \Omega} \leq C h .
$$

Proof. We get the following error equation by combining (2.1) with (3.14), for all $\left(v_{h}, q_{h}\right) \in$ $\left(\mathrm{NCP}_{1}, P_{1}\right)$

$$
\begin{aligned}
& B\left(\left(u-u_{h}, p-p_{h}\right) ;\left(v_{h}, q_{h}\right)\right)+b\left(u-u_{h}, u, v_{h}\right)+b\left(u_{h}, u-u_{h}, v_{h}\right)-\sum_{K_{j}}\left\langle\frac{\partial u}{\partial n}, v_{h}\right\rangle_{j} \\
& \quad+\sum_{K_{j}}\left\langle v_{h} \cdot n, p\right\rangle_{j}=\sum_{K_{j}} \tau_{K_{j}}\left(\nabla p, \nabla q_{h}\right)_{K_{j}}-\sum_{K_{j}} \tau_{K_{j}}\left(f, \nabla q_{h}\right)_{K_{j}} .
\end{aligned}
$$

With (4.3), (4.21) can be rewritten as

$$
\begin{gathered}
B\left(\left(e_{h}, \eta_{h}\right) ;\left(v_{h}, q_{h}\right)\right)+b\left(u-R_{h}(u, p)+e_{h}, u, v_{h}\right)+b\left(u_{h}, u-R_{h}(u, p)+e_{h}, v_{h}\right) \\
-\sum_{K_{j}}\left\langle\frac{\partial u}{\partial n}, v_{h}\right\rangle_{j}+\sum_{K_{j}}\left\langle v_{h} \cdot n, p\right\rangle_{j}=-\sum_{K_{j}} \tau_{K_{j}}\left(f, \nabla q_{h}\right)_{K_{j}{ }^{\prime}}
\end{gathered}
$$

where $e_{h}=R_{h}(u, p)-u_{h}$ and $\eta_{h}=Q_{h}(u, p)-p_{h}$. 
From Theorem 3.5 and (4.22), we get that

$$
\begin{aligned}
\left\|\eta_{h}\right\|_{0, \Omega} \leq & \beta^{-1} \sup _{0 \neq\left(v_{h}, q_{h}\right) \in\left(\mathrm{NCP}_{1}, P_{1}\right)} \frac{\left|B\left(\left(e_{h}, \eta_{h}\right) ;\left(v_{h}, q_{h}\right)\right)\right|}{\left\|v_{h}\right\|_{1, h}+\left\|q_{h}\right\|_{0, \Omega}} \\
\leq & \beta^{-1} \sup _{0 \neq\left(v_{h}, q_{h}\right) \in\left(\mathrm{NCP}_{1}, P_{1}\right)} \frac{1}{\left\|v_{h}\right\|_{1, h}+\left\|q_{h}\right\|_{0, \Omega}} \\
& \times\left(\left|b\left(u-R_{h}(u, p)+e_{h}, u, v_{h}\right)\right|+\left|\sum_{K_{j}} \tau_{K_{j}}\left(f, \nabla q_{h}\right)_{K_{j}}\right|\right. \\
& \left.\quad+\left|b\left(u_{h}, u-R_{h}(u, p)+e_{h}, v_{h}\right)\right|+\left|\sum_{K_{j}}\left\langle\frac{\partial u}{\partial n}, v_{h}\right\rangle_{j}\right|+\left|\sum_{K_{j}}\left\langle v_{h} \cdot n, p\right\rangle_{j}\right|\right) .
\end{aligned}
$$

Again, with (2.13), Theorem 2.1, inverse inequality, and Lemma 4.1, we have

$$
\begin{aligned}
&\left|b\left(u-R_{h}(u, p), u, v_{h}\right)\right|+\left|b\left(u_{h}, u-R_{h}(u, p), v_{h}\right)\right| \\
& \leq c_{0} \gamma_{0}\left(|u|_{1, \Omega}+\left\|u_{h}\right\|_{1, h}\right)\left\|u-R_{h}(u, p)\right\|_{1, h}\left\|v_{h}\right\|_{1, h} \leq C h\left\|v_{h}\right\|_{1, h^{\prime}} \\
&\left|b\left(e_{h}, u, v_{h}\right)\right|+\left|b\left(u_{h}, e_{h}, v_{h}\right)\right| \leq c_{0} \gamma_{0}\left(|u|_{1, \Omega}+\left\|u_{h}\right\|_{1, h}\right)\left\|e_{h}\right\|_{1, h}\left\|v_{h}\right\|_{1, h^{\prime}} \\
& \sum_{K_{j}} \tau_{K_{j}}\left|\left(f, \nabla q_{h}\right)_{K_{j}}\right| \leq \sum_{K_{j}} \tau_{K_{j}}\|f\|_{0, K_{j}}\left\|\nabla q_{h}\right\|_{0, K_{j}} \\
& \leq \sum_{K_{j}} \tilde{C} h_{K_{j}}^{2}\|f\|_{0, K_{j}}\left\|\nabla q_{h}\right\|_{0, K_{j}} \\
& \leq C(v) h\|f\|_{0, \Omega}\left\|q_{h}\right\|_{0, \Omega^{\prime}}
\end{aligned}
$$

and using the similar arguments as for (4.17)-(4.18) yields

$$
\left|\sum_{K_{j}}\left\langle\frac{\partial u}{\partial n}, v_{h}\right\rangle_{j}\right|+\left|\sum_{K_{j}}\left\langle v_{h} \cdot n, p\right\rangle_{j}\right| \leq C h^{2}\left(\|A u\|_{0, \Omega}+|p|_{1, \Omega}\right)\left\|v_{h}\right\|_{1, h} .
$$

Combining (4.23)-(4.27) with Theorem 3.5, we arrive at

$$
\left\|\eta_{h}\right\|_{0, \Omega} \leq C h+2 c_{0} \gamma_{0} \frac{\gamma_{0}+v^{1 / 2}}{v}\|f\|_{0, \Omega}\left\|e_{h}\right\|_{1, h} .
$$


Choosing $\left(v_{h}, q_{h}\right)=\left(e_{h}, \eta_{h}\right)$ in $(4.22)$, we obtain that

$$
\begin{aligned}
B\left(\left(e_{h}, \eta_{h}\right) ;\left(e_{h}, \eta_{h}\right)\right)+b\left(e_{h}, u, e_{h}\right)+\sum_{K_{j}} \tau_{K_{j}}\left(f, \nabla \eta_{h}\right)_{K_{j}} \\
=-b\left(u-R_{h}(u, p), u, e_{h}\right)-b\left(u_{h}, u-R_{h}(u, p), e_{h}\right) \\
\quad+\sum_{K_{j}}\left\langle\frac{\partial u}{\partial n}, v_{h}\right\rangle_{j}-\sum_{K_{j}}\left\langle e_{h} \cdot n, p\right\rangle_{j} .
\end{aligned}
$$

Using (2.13), (2.17), Theorem 2.1, and Lemma 3.3, we get

$$
\begin{aligned}
B\left(\left(e_{h}, \eta_{h}\right) ;\left(e_{h}, \eta_{h}\right)\right)-b\left(e_{h}, u, e_{h}\right) & =\left\|e _ { h } \left|\left\|_{h}^{2}+\right\| \eta_{h}\left\|_{h}^{2}-c_{0} \gamma_{0}|u|_{1}\right\| e_{h} \|_{1, h}^{2}\right.\right. \\
& \geq v\left\|e_{h}\right\|_{1, h}^{2}-c_{0} \gamma_{0}|u|_{1}\left\|e_{h}\right\|_{1, h}^{2} \\
& \geq v\left(1-c_{0} \gamma_{0}^{2} v^{-2}\|f\|_{0}\right)\left\|e_{h}\right\|_{1, h}^{2}>0 .
\end{aligned}
$$

Combining (4.24)-(4.28) with (4.29) yields:

$$
\left\|e_{h}\right\|_{1, h} \leq C h .
$$

From (4.28) and (4.31), we obtain that $\left\|\eta_{h}\right\|_{0, \Omega} \leq C h$. Furthermore, we finish the proof by combining triangles inequality with Lemma 4.1, (4.28), and (4.31).

Theorem 4.3. Let $(u, p)$ and $\left(u_{h}, p_{h}\right)$ be the solutions of (2.1) and (3.14), respectively, then we have

$$
\left\|u-u_{h}\right\|_{0, \Omega} \leq C h^{2}\left(\|A u\|_{0, \Omega}+|p|_{1, \Omega}\right) .
$$

Proof. Using the duality argument for a linearized stationary Navier-Stokes problem; for some given $g \in Y$ and the solution $(u, p)$ of $(2.1)$, defining $(\Phi, \Psi) \in(X, M)$ by

$$
\begin{gathered}
-v \Delta \Phi+\nabla \Psi+\widetilde{B}(u, \Phi)-B(u, \Phi)=g, \quad \text { in } \Omega, \\
\operatorname{div} \Phi=0 \quad \text { in } \Omega, \\
\left.u\right|_{\partial \Omega}=0 \quad \text { on } \partial \Omega,
\end{gathered}
$$

where $\widetilde{B}(u, \Phi)$ is defined as $\langle v, \widetilde{B}(u, \Phi)\rangle_{X \times X^{\prime}}=b(v, u, \Phi)$, for all $v \in X$; multiplying (4.33) and (4.34) by $v \in X$ and $q \in M$, respectively; integrating over $\Omega$, from (2.8)-(2.11), it is easily to see that the bilinear form $a(\Phi, v)-d(v, \Psi)+d(\Phi, q)$ is continuity and $X \times M$ coercive, by using the Lax-Milgram's Lemma, (4.33)-(4.35) have a unique solution $(\Phi, \Psi)$. 
Multiplying (4.33) and (4.34) by $\Phi$ and $\Psi$, respectively, using (2.13) and Theorem 2.1, we have

$$
\begin{aligned}
v|\Phi|_{1, \Omega}^{2}-b(\Phi, u, \Phi) & \geq v|\Phi|_{1, \Omega}^{2}-c_{0} r_{0}|u|_{1, \Omega}|\Phi|_{1, \Omega}^{2} \\
& \geq v\left(1-c_{0} r_{0}^{2} v^{-2}\|f\|_{0, \Omega}\right)|\Phi|_{1, \Omega}^{2}>0 .
\end{aligned}
$$

On the other hand, estimating the right term yields

$$
(\Phi, g) \leq\|\Phi\|_{0, \Omega}\|g\|_{0, \Omega} \leq \gamma_{0}|\Phi|_{1, \Omega}\|g\|_{0, \Omega}
$$

By using (4.36) and (4.37), we arrive at

$$
|\Phi|_{1, \Omega} \leq C\|g\|_{0, \Omega}
$$

Setting $\Psi=0$ and taking the scalar product of (4.33) with $A \Phi$ in $Y$ yields

$$
v\|A \Phi\|_{0}^{2}+b(A \Phi, u, \Phi)-b(u, \Phi, A \Phi)=(g, A \Phi) .
$$

Using the Gagliardo-Nirenberg inequality yields

$$
\begin{gathered}
\|v\|_{L^{4}}^{2} \leq C\|v\|_{0, \Omega}|v|_{1, \Omega}, \quad \forall v \in X \\
\|\nabla v\|_{L^{4}}^{2} \leq C\|A v\|_{0, \Omega}|v|_{1, \Omega}, \quad \forall v \in H^{2}(\Omega)^{2} \cap X .
\end{gathered}
$$

With the help of the Agmon's inequality, we have

$$
\|v\|_{L^{\infty}}^{2} \leq C\|v\|_{0, \Omega}\|A v\|_{0, \Omega}, \quad \forall v \in H^{2}(\Omega)^{2} \cap X
$$

Furthermore, the following estimates are hold:

$$
\begin{gathered}
b(A \Phi, u, \Phi) \leq C\|A \Phi\|_{0, \Omega}\left(\|\nabla u\|_{L^{4}}\|\Phi\|_{L^{4}}+\|u\|_{L^{\infty}}\|\nabla \Phi\|_{0, \Omega}\right) \\
\left.\leq \frac{v}{4}\|A \Phi\|_{0, \Omega}^{2}+C v^{-1}\|A u\|_{0, \Omega}^{2}\|\nabla \Phi\|_{0, \Omega}^{2}\right), \\
\left.b(u, \Phi, A \Phi) \leq \frac{v}{4}\|A \Phi\|_{0, \Omega}^{2}+C v^{-1}\|A u\|_{0, \Omega}^{2}\|\nabla \Phi\|_{0, \Omega}^{2}\right), \\
(g, A \Phi) \leq \frac{v}{4}\|A \Phi\|_{0, \Omega}^{2}+C v^{-1}\|g\|_{0, \Omega} .
\end{gathered}
$$

Combining above inequalities with (4.38) and (4.39), we arrive at

$$
\|A \Phi\|_{0, \Omega}^{2} \leq c v^{-2}\left(1+\|A u\|_{0, \Omega}^{2}\right)\|g\|_{0, \Omega}^{2}
$$


Applying the continuous Inf-Sup condition (2.8) yields

$$
|\Psi|_{1, \Omega} \leq c \mathcal{v}\|A \Phi\|_{0, \Omega}+c\|A u\|_{0, \Omega}|\Phi|_{1, \Omega}+c\|g\|_{0, \Omega}
$$

Combining (4.43)-(4.44) with (2.18), (4.38), we arrive at

$$
\|A \Phi\|_{0, \Omega}+|\Psi|_{1, \Omega} \leq C\|g\|_{0, \Omega}
$$

Taking $g=R_{h}(u, p)-u_{h}$, multiplying (4.33) and (4.34) by $R_{h}(u, p)-u_{h}$ and $Q_{h}(u, p)-p_{h}$, respectively, using (4.1) yields

$$
\begin{aligned}
\left\|R_{h}(u, p)-u_{h}\right\|_{0, \Omega}^{2} \\
=B\left(\left(R_{h}(u, p)-u_{h}, Q_{h}(u, p)-p_{h}\right) ;(\Phi, \Psi)\right)+b\left(u, R_{h}(u, p)-u_{h}, \Phi\right) \\
\quad-\sum_{K_{j}} \tau_{K_{j}}\left(\nabla\left(Q_{h}(u, p)-p_{h}\right), \nabla \Psi\right)_{K_{j}}+\sum_{K_{j}}\left\langle\Psi,\left(R_{h}(u, p)-u_{h}\right) \cdot n,\right\rangle_{j} \\
\quad+b\left(R_{h}(u, p)-u_{h}, u, \Phi\right)-\sum_{K_{j}}\left\langle\frac{\partial \Phi}{\partial n}, R_{h}(u, p)-u_{h}\right\rangle_{j} .
\end{aligned}
$$

Setting $\left(v_{h}, q_{h}\right)=\left(\Phi_{I}, \Psi_{I}\right)$ in $(4.22)$, and using (4.46), we obtain that

$$
\begin{aligned}
& \left\|R_{h}(u, p)-u_{h}\right\|_{0, \Omega}^{2} \\
& =B\left(\left(R_{h}(u, p)-u_{h}, Q_{h}(u, p)-p_{h}\right) ;\left(\Phi-\Phi_{I}, \Psi-\Psi_{I}\right)\right) \\
& -\sum_{K_{j}} \tau_{K_{j}}\left(\nabla\left(Q_{h}(u, p)-p_{h}\right), \nabla \Psi\right)_{K_{j}}+b\left(u-u_{h}, R_{h}(u, p)-u_{h}, \Phi\right)+\sum_{K_{j}}\left\langle\frac{\partial u}{\partial n}, v_{h}\right\rangle_{j} \\
& +b\left(u-R_{h}(u, p), u, \Phi_{I}\right)+b\left(R_{h}(u, p)-u_{h}, u, \Phi-\Phi_{I}\right)+b\left(u_{h}, u-R_{h}(u, p), \Phi-\Phi_{I}\right) \\
& +b\left(u_{h}, R_{h}(u, p)-u_{h}, \Phi-\Phi_{I}\right)-b\left(u_{h}, u-R_{h}(u, p), \Phi\right)+\sum_{K_{j}}\left\langle\Psi,\left(R_{h}(u, p)-u_{h}\right) \cdot n,\right\rangle_{j} \\
& -\sum_{K_{j}} \tau_{K_{j}}\left(f, \nabla \Psi_{I}\right)_{K_{j}}-\sum_{K_{j}}\left\langle\frac{\partial \Phi}{\partial n}, R_{h}(u, p)-u_{h}\right\rangle_{j}-\sum_{K_{j}}\left\langle v_{h} \cdot n_{,} p\right\rangle_{j} .
\end{aligned}
$$


We now estimate the right terms of (4.47). Thanks to (3.3); Theorems 2.1, 3.4-3.5, and 4.2; inverse inequality; Lemma 4.1; we know that

$$
\begin{aligned}
& B\left(\left(R_{h}(u, p)-u_{h}, Q_{h}(u, p)-p_{h}\right) ;\left(\Phi-\Phi_{I}, \Psi-\Psi_{I}\right)\right) \\
& \leq C\left(\left\|R_{h}(u, p)-u_{h}\right\|_{1, h}+\left\|Q_{h}(u, p)-p_{h}\right\|_{0, \Omega}\right)\left(\left\|\Phi-\Phi_{I}\right\|_{1, h}+\left\|\Psi-\Psi_{I}\right\|_{0, \Omega}+h|\Psi|_{1, \Omega}\right) \\
& \leq C h^{2}\left(\|A u\|_{0, \Omega}+|p|_{1, \Omega}\right)\left(\|A \Phi\|_{0, \Omega}+|\Psi|_{1, \Omega}\right), \\
& b\left(R_{h}(u, p)-u_{h}, u, \Phi-\Phi_{I}\right)+b\left(u_{h}, R_{h}(u, p)-u_{h}, \Phi-\Phi_{I}\right) \\
& \leq 2 c_{0}\left(|u|_{1, \Omega}+\left\|u_{h}\right\|_{1, h}\right)\left\|R_{h}(u, p)-u_{h}\right\|_{1, h}\left\|\Phi-\Phi_{I}\right\|_{1, h} \\
& \leq C h^{2}\left(\|A u\|_{0, \Omega}+|p|_{1, \Omega}\right)\|A \Phi\|_{0, \Omega} \\
& \sum_{K_{j}} \tau_{K_{j}}\left(\nabla\left(Q_{h}(u, p)-p_{h}\right), \nabla \Psi\right)_{K_{j}} \leq \sum_{K_{j}} \tilde{C} h_{K}^{2}\left\|\nabla\left(Q_{h}(u, p)-p_{h}\right)\right\|_{0, K_{j}}\|\nabla \Psi\|_{0, K_{j}} \\
& \leq C h^{2}\left(\|A u\|_{0, \Omega}+|p|_{1}\right)|\Psi|_{1, \Omega} \\
& b\left(u-R_{h}(u, p), u, \Phi_{I}\right) \leq C\left\|u-R_{h}(u, p)\right\|_{0, \Omega}\|A u\|_{0, \Omega}\left|\Phi_{I}\right|_{1, h} \\
& \leq C h^{2}\left(\|A u\|_{0, \Omega}+|p|_{1, \Omega}\right)|\Phi|_{1, \Omega} \\
& b\left(u-u_{h}, R_{h}(u, p)-u_{h}, \Phi\right) \leq c_{0}\left\|u-u_{h}\right\|_{1, h}\left\|R_{h}(u, p)-u_{h}\right\|_{1, h}|\Phi|_{1, \Omega} \\
& \leq C h^{2}\left(\|A u\|_{0, \Omega}+|p|_{1}\right)|\Phi|_{1, \Omega} \\
& b\left(u_{h}, u-R_{h}(u, p), \Phi\right) \leq C\left\|u_{h}\right\|_{1, h}\left\|u-R_{h}(u, p)\right\|_{0, \Omega}\|A \Phi\|_{0, \Omega}, \\
& \sum_{K_{j}} \tau_{K_{j}}\left(f, \nabla \Psi_{I}\right)_{K_{j}} \leq \sum_{K_{j}} \tilde{C} h_{K_{j}}^{2}\|f\|_{0, K_{j}}\left\|\nabla \Psi_{I}\right\|_{0, K_{j}} \leq C h^{2}\|f\|_{0, \Omega}|\Psi|_{1, \Omega} \\
& b\left(u_{h}, u-R_{h}(u, p), \Phi-\Phi_{I}\right) \leq c_{0}\left\|u_{h}\right\|_{1, h}\left\|u-R_{h}(u, p)\right\|_{1, h}\left\|\Phi-\Phi_{I}\right\|_{1, h} \\
& \leq C h^{2}\left(\|A u\|_{0, \Omega}+|p|_{1, \Omega}\right)\|A \Phi\|_{0, \Omega} .
\end{aligned}
$$

Applying the argument used for (4.18)-(4.21), (4.28) gives

$$
\begin{gathered}
\sum_{K_{j}}\left\langle\frac{\partial \Phi}{\partial n}, R_{h}(u, p)-u_{h}\right\rangle_{j}+\sum_{K_{j}}\left\langle\Psi,\left(R_{h}(u, p)-u_{h}\right) \cdot n,\right\rangle_{j} \\
\leq C h^{2}\left(\|A u\|_{0, \Omega}+|p|_{1, \Omega}\right)\left(\|A \Phi\|_{0, \Omega}+|\Psi|_{1, \Omega}\right), \\
\sum_{K_{j}}\left\langle\frac{\partial u}{\partial n}, \Phi_{I}\right\rangle+\sum_{K_{j}}\left\langle\Phi_{I} \cdot n, p\right\rangle_{j} \leq C h^{2}\left(\|A u\|_{0, \Omega}+\|p\|_{1, \Omega}\right)\left\|\Phi_{I}\right\|_{1, h} \\
\leq C h^{2}\left(\|A u\|_{0, \Omega}+\|p\|_{1, \Omega}\right)\left\|\Phi_{I}\right\|_{1, \Omega} .
\end{gathered}
$$


Combining above inequalities, Theorems 2.1, 3.5, (4.46), and (4.51), we get that

$$
\begin{aligned}
& \left\|R_{h}(u, p)-u_{h}\right\|_{0, \Omega}^{2} \\
& \quad \leq C h^{2}\left(\|A u\|_{0, \Omega}+|p|_{1, \Omega}\right)\left(\|A \Phi\|_{0, \Omega}+|\Psi|_{1, \Omega}\right)+C\left\|u_{h}\right\|_{1, h}\left\|u-R_{h}(u, p)\right\|_{0, \Omega}\|A \Phi\|_{0, \Omega} \\
& \quad \leq C h^{2}\left(\|A u\|_{0, \Omega}+|p|_{1, \Omega}\right)\left\|R_{h}(u, p)-u_{h}\right\|_{1, \Omega}+C \frac{\gamma_{0}+v^{1 / 2}}{v}\|f\|_{0, \Omega}\left\|u-R_{h}(u, p)\right\|_{0, \Omega}^{2} .
\end{aligned}
$$

Choosing the appropriate $v, \Omega$ and $f$ such that $1-C\left(\left(\gamma_{0}+v^{1 / 2}\right) / v\right)\|f\|_{0}>0$, then we have

$$
\left\|R_{h}(u, p)-u_{h}\right\|_{0, \Omega} \leq C h^{2}\left(\|A u\|_{0, \Omega}+\|p\|_{1, \Omega}\right)
$$

By applying the triangles inequality, Lemma 4.1 and (4.51), we finish the proof.

Lemma 4.4. Under the assumptions of Theorem 4.2, the following estimate about $u-u_{h}$ in meshdependent norm

$$
\left\|\left|u-u_{h}\right|\right\|_{h} \leq C h
$$

holds, where $u$ and $u_{h}$ are the solution of problem (2.1) and (3.14), respectively.

Proof. According to the definition of $\||\cdot|\|_{h}, u_{h} \in X_{h}$, with Theorems 2.1 and 4.2, inverse and local trace inequalities (3.21), we have

$$
\begin{aligned}
\left\|u-u_{h} \mid\right\|_{h}^{2} & =v\left\|u-u_{h} \mid\right\|_{1, h}^{2}+\sum_{E \in \Gamma_{h}} \tau_{E}\left\|v \partial_{n}\left(u-u_{h}\right)\right\|_{0, E}^{2} \\
& =v\left\|u-u_{h}\right\|_{1, h}^{2}+\sum_{E \in \Gamma_{h}} \frac{h_{e}}{12 v}\left\|v \partial_{h}\left(u-u_{h}\right)\right\|_{0, E}^{2} \\
& \leq C h^{2}+C \sum_{E \in \Gamma_{h}} h_{e}\left[h_{K_{j}}^{-1}\left\|\nabla\left(u-u_{h}\right) \cdot n\right\|_{0, K_{j}}^{2}+h_{K_{j}}\left|\nabla\left(u-u_{h}\right) \cdot n\right|_{1, K_{j}}^{2}\right] \\
& \leq C h^{2} .
\end{aligned}
$$

\section{Numerical Validations}

In this section, we provide two numerical examples to illustrate the theoretical analysis of the method (3.14). In all experiments, we consider the domain $\Omega$ to be the square $[0,1] \times[0,1]$. The mesh consists of triangular elements that are obtained by dividing $\Omega$ into subsquares of equal size and then drawing the diagonal in each sub-square, see Figure 1. The software Freefem++, developed by Hecht et al. [23], is used in our experiments. 


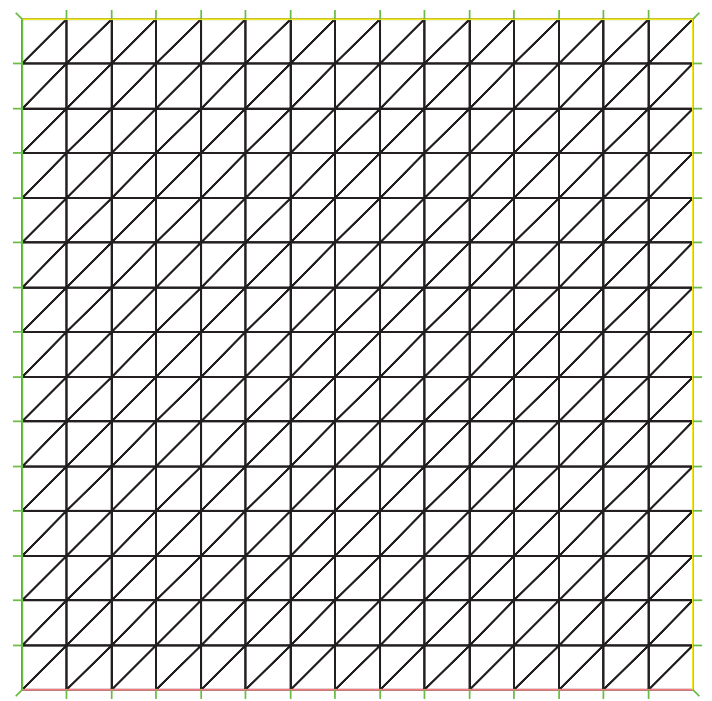

Figure 1: Uniform triangulation of $\Omega$ into triangulars.

Table 1: Numerical results for enriched multiscale method with $\mathrm{NCP}_{1}-P_{1}$ element.

\begin{tabular}{lcccccc}
\hline $1 / h$ & $\left\|p_{h}-\tilde{p}\right\|_{0} /\|\tilde{p}\|_{0}$ & $\left\|u_{h}-\tilde{u}\right\|_{0} /\|\tilde{u}\|_{0}$ & $\left|u_{h}-\tilde{u}\right|_{1} /|\tilde{u}|_{1}$ & $p_{L^{2}}$ rate & $u_{L^{2}}$ rate & $u_{H^{1}}$ rate \\
\hline 20 & 0.160667 & 0.15627 & 0.816235 & & & \\
25 & 0.126534 & 0.10132 & 0.665410 & 1.0703 & 1.9418 & 0.9155 \\
30 & 0.105785 & 0.0708881 & 0.556179 & 0.9823 & 1.9591 & 0.9835 \\
35 & 0.083427 & 0.0523941 & 0.478261 & 1.5403 & 1.9611 & 0.9791 \\
40 & 0.0667358 & 0.0405399 & 0.420987 & 1.6717 & 1.9209 & 0.9552 \\
\hline
\end{tabular}

Table 2: Numerical results for PPM in [6] with $\mathrm{NCP}_{1}-P_{1}$ element.

\begin{tabular}{lcccccc}
\hline $1 / h$ & $\left\|p_{h}-p\right\|_{0} /\|\tilde{p}\|_{0}$ & $\left\|u_{h}-u\right\|_{0} /\|u\|_{0}$ & $\left\|u_{h}-u\right\|_{1} /\|u\|_{1}$ & $p_{L^{2}}$ rate & $u_{L^{2}}$ rate & $u_{H^{1}}$ rate \\
\hline 20 & 0.208238 & 0.0182227 & 0.12718 & 1.8797 & 1.9629 & 0.9963 \\
25 & 0.136834 & 0.0117122 & 0.101772 & 1.8818 & 1.9809 & 0.9988 \\
30 & 0.0972068 & 0.0081486 & 0.0848213 & 1.8754 & 1.9898 & 0.9993 \\
35 & 0.0727087 & 0.00599297 & 0.0727009 & 1.8837 & 1.9932 & 1.0002 \\
40 & 0.056478 & 0.00459177 & 0.0636082 & 1.8918 & 1.9945 & 1.0006 \\
\hline
\end{tabular}

\subsection{An Analytical Solution: Convergence Validation}

For this test, our purpose is to verify the theoretical analysis which has been established in the previous section by setting the viscosity coefficient $v=1$ and $f$ is given by the exact solution

$$
\begin{gathered}
u_{1}=10 x^{2}(x-1)^{2} y(y-1)(2 y-1), \\
u_{2}=-10 x(x-1)(2 x-1) y^{2}(y-1)^{2}, \\
p=10(2 x-1)(2 y-1) .
\end{gathered}
$$

In our numerical validation, The experimental rates of convergence with respect to the mesh size $h$ are calculated by the formula $\left(\log \left(E_{i} / E_{i+1}\right)\right) /\left(\log \left(h_{i} / h_{i+1}\right)\right)$, where $E_{i}$ and $E_{i+1}$ are the relative errors corresponding to the meshes of sizes $h_{i}$ and $h_{i+1}$. 
Table 3: Numerical results for SGM with $P_{1} b-P_{1}$ element.

\begin{tabular}{lcccccc}
\hline $1 / h$ & $\left\|p_{h}-p\right\|_{0} /\|p\|_{0}$ & $\left\|u_{h}-u\right\|_{0} /\|u\|_{0}$ & $\left\|u_{h}-u\right\|_{1} /\|u\|_{1}$ & $p_{L^{2}}$ rate & $u_{L^{2}}$ rate & $u_{H^{1}}$ rate \\
\hline 20 & 0.194855 & 0.0167771 & 0.120396 & 1.9352 & 2.0150 & 1.0040 \\
25 & 0.127314 & 0.010703 & 0.0962298 & 1.9073 & 2.0144 & 1.0041 \\
30 & 0.0903239 & 0.00741503 & 0.080144 & 1.8827 & 2.0130 & 1.0033 \\
35 & 0.0675341 & 0.00543371 & 0.068793 & 1.8863 & 2.0168 & 0.9907 \\
40 & 0.0527341 & 0.00416312 & 0.059691 & 1.8532 & 1.9947 & 1.0628 \\
\hline
\end{tabular}

Table 4: Numerical results for LGIM in $[4,7,19]$ with $\mathrm{NCP}_{1}-P_{1}$ element.

\begin{tabular}{lcccccc}
\hline $1 / h$ & $\left\|p_{h}-p\right\|_{0} /\|p\|_{0}$ & $\left\|u_{h}-u\right\|_{0} /\|u\|_{0}$ & $\left\|u_{h}-u\right\|_{1} /\|u\|_{1}$ & $p_{L^{2}}$ rate & $u_{L^{2}}$ rate & $u_{H^{1}}$ rate \\
\hline 20 & 0.263248 & 0.0186335 & 0.127546 & 1.7919 & 2.0088 & 1.0010 \\
25 & 0.176286 & 0.0118944 & 0.102010 & 1.7970 & 2.0117 & 1.0012 \\
30 & 0.128021 & 0.00824161 & 0.0849864 & 1.7547 & 2.0122 & 1.0014 \\
35 & 0.0978302 & 0.00604517 & 0.0728229 & 1.7448 & 2.0106 & 1.0020 \\
40 & 0.0776455 & 0.00462309 & 0.0637016 & 1.7305 & 2.0085 & 1.0022 \\
\hline
\end{tabular}

Table 5: CPU time for solving the steady Navier-Stokes equations with different mesh sizes.

\begin{tabular}{lccccc}
\hline $1 / h$ & 20 & 25 & 30 & 35 & 40 \\
\hline Our method with $\mathrm{NCP}_{1}-P_{1}$ & 1.6324 & 3.286 & 5.975 & 8.792 & 14.088 \\
PPM in [6] with $\mathrm{NCP}_{1}-P_{1}$ & 1.875 & 3.594 & 6.406 & 10.891 & 17.453 \\
LGIM in [10] with $\mathrm{NCP}_{1}-P_{1}$ & 1.844 & 3.547 & 6.391 & 10.593 & 17.047 \\
SGM with $P_{1} b-P_{1}$ & 3.031 & 6.078 & 11.375 & 21.106 & 35.020 \\
\hline
\end{tabular}

In order to show the efficiency of the enriched multiscale method, we compare the numerical results obtained by using different methods, which are shown in Tables 1, 2, 3, and 4 . The compared methods include the pressure projection method (PPM) in [6], the local Gauss integration method (LGIM) in $[7,10,19]$, and the standard Galerkin method (SGM) with MINI element (see [1]), respectively. From these tables, we can see that the stabilized multiscale method has good precision for pressure, and the precision of velocity, worse than other methods. Table 5 explains the CPU times that needed for solving the steady NavierStokes equations in different mesh sizes. From these data, we know that our method takes less time than other methods. Furthermore, from Tables 1-4, we can see that the numerical results reproduce the established theoretical analysis and show an $\mathcal{O}(h)$ order of convergence for $\left|u-u_{h}\right|_{1, \Omega}$ and $\left\|p-p_{h}\right\|_{0, \Omega}$, and an $\mathcal{O}\left(h^{2}\right)$ convergence for $\left\|u-u_{h}\right\|_{0, \Omega}$.

\subsection{Lid-Driven Cavity Problem}

In this test, we consider the incompressible lid-driven cavity flow problem defined on the unit square. Setting $f=0$ and the boundary condition $u=0$ on $[\{0\} \times(0,1)] \cup[(0,1) \times\{0\}] \cup[\{1\} \times$ $(0,1)]$ and $u=(1,0)^{T}$ on $(0,1) \times\{1\}$, see Figure 2 . The mesh consists of triangular element and the mesh size $h=1 / 50$.

Figure 3 shows the pressure contours at different Reylond numbers, where the stopping criterion $\left\|u_{h}^{n+1}-u_{h}^{n}\right\|_{0, \Omega} /\left\|u_{h}^{n+1}\right\|_{0, \Omega} \leq 10^{-6}$ is employed, where $u_{h}^{n+1}$ is the approximation of $u_{h}$ at the $n+1$ Newton iterative. From Figure 3, we can see that the oscillations are absented for the pressure isovalues by the $\mathrm{NCP}_{1}-P_{1}$ approximations, and compared with the results given in [24], we can see that our method has the effect to stabilized flow field (see Figure 4). 


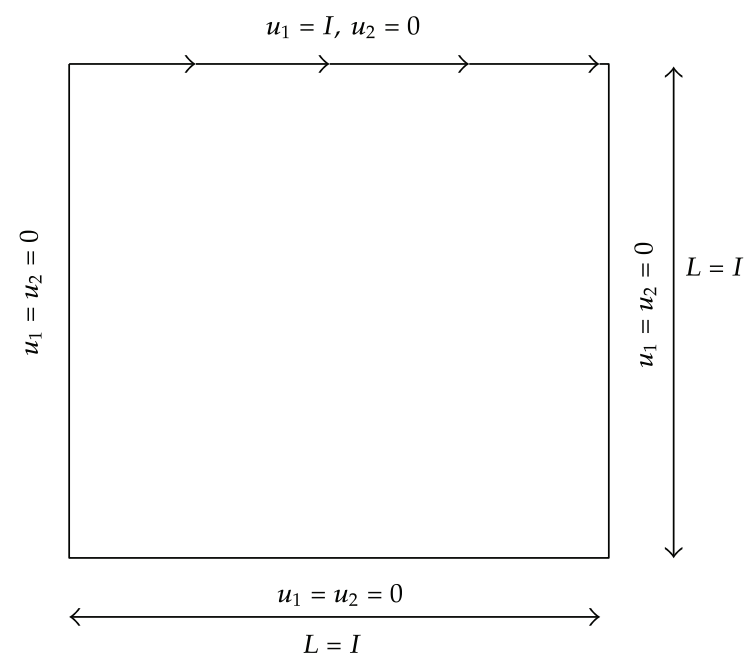

Figure 2: Lid-driven cavity flow.

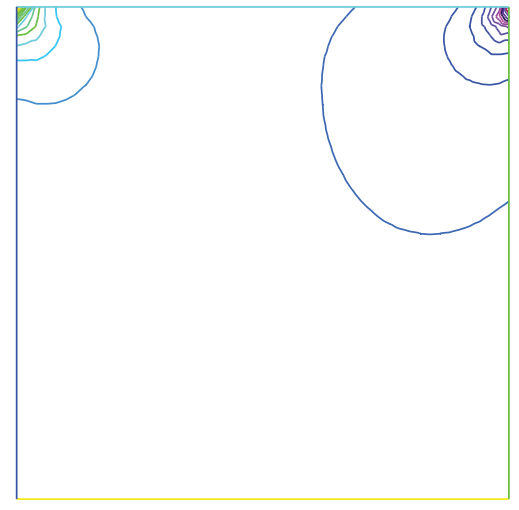

(a)

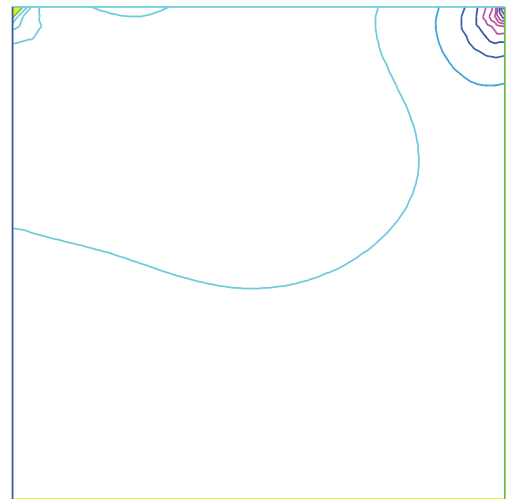

(b)

Figure 3: Pressure contours of the driven cavity by using the stabilized multiscale method with $\mathrm{NCP}_{1}-P_{1}$ at different Reynolds numbers. (a) $\operatorname{Re}=1$, (b) $\operatorname{Re}=100$.

In this sense, we say that the stabilized multiscale nonconforming finite element method is effective for the stationary Navier-Stokes problem.

\section{Conclusion}

In this paper we have derived a theoretical analysis of enriched multiscale nonconforming finite element method for the steady incompressible Navier-Stokes equations. The analysis has extended the work in [16] from the linear problem to the nonlinear problem. The discretization uses nonconforming and conforming piecewise linear finite elements for velocity and pressure over triangles elements, respectively. Numerical tests show that this stabilized method is computationally efficient, and it can be performed locally at the element 


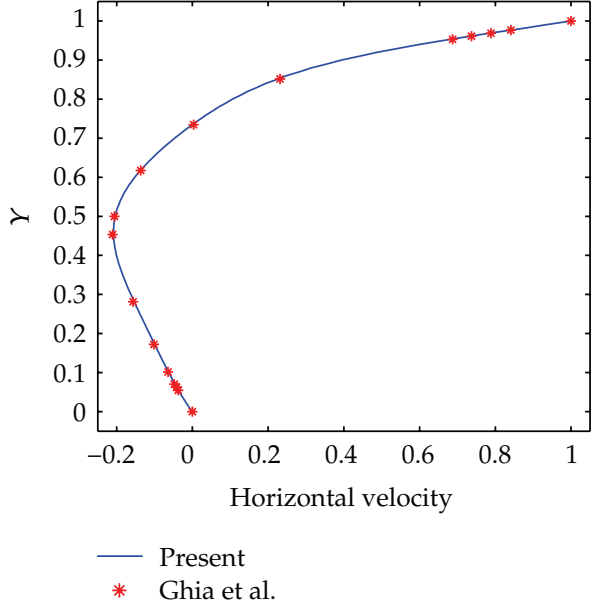

(a)

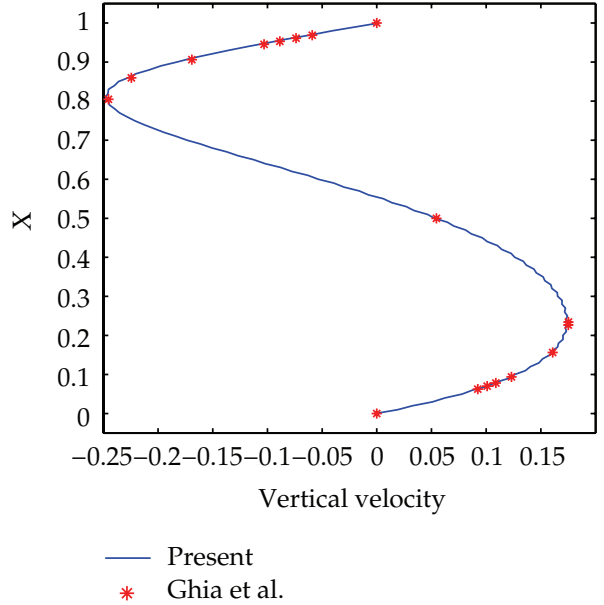

(b)

Figure 4: The computed velocity profiles through the geometric center at $\operatorname{Re}=100$. (a) Horizontal velocity, (b) vertical velocity.

level with minimal additional cost; at the same time, our numerical results obtained are in good agreement with the established theoretical results.

\section{Acknowledgments}

This work was supported by the Natural Science Foundation of China (no. 11126117) and the Doctor Fund of Henan Polytechnic University (no. 648689).

\section{References}

[1] V. Girault and P.-A. Raviart, Finite Element Method for Navier-Stokes equations: Theory and Algorithms, vol. 5, Springer, Berlin, Germany, 1986.

[2] R. Codina and J. Blasco, "Analysis of a pressure-stabilized finite element approximation of the stationary Navier-Stokes equations," Numerische Mathematik, vol. 87, no. 1, pp. 59-81, 2000.

[3] Y. He, "A fully discrete stabilized finite-element method for the time-dependent Navier-Stokes problem," IMA Journal of Numerical Analysis, vol. 23, no. 4, pp. 665-691, 2003.

[4] J. Li and Z. Chen, "A new local stabilized nonconforming finite element method for the Stokes equations," Computing, vol. 82, no. 2-3, pp. 157-170, 2008.

[5] G. R. Barrenechea and F. Valentin, "An unusual stabilized finite element method for a generalized Stokes problem," Numerische Mathematik, vol. 92, no. 4, pp. 653-677, 2002.

[6] R. Becker and P. Hansbo, "A simple pressure stabilization method for the Stokes equation," Communications in Numerical Methods in Engineering with Biomedical Applications, vol. 24, no. 11, pp. 1421-1430, 2008.

[7] P. Bochev and M. Gunzburger, "An absolutely stable pressure-Poisson stabilized finite element method for the Stokes equations," SIAM Journal on Numerical Analysis, vol. 42, no. 3, pp. 1189-1207, 2004.

[8] T. Zhang and Y. N. He, "Fully discrete finite element method based on pressure stabilization for the transient Stokes equations," Mathematics and Computers in Simulation, vol. 82, pp. 1496-1515, 2012.

[9] J. Li and Y. He, "A stabilized finite element method based on two local Gauss integrations for the Stokes equations," Journal of Computational and Applied Mathematics, vol. 214, no. 1, pp. 58-65, 2008. 
[10] J. Li, Y. He, and Z. Chen, "Performance of several stabilized finite element methods for the Stokes equations based on the lowest equal-order pairs," Computing, vol. 86, no. 1, pp. 37-51, 2009.

[11] J. Li, Y. He, and Z. Chen, "A new stabilized finite element method for the transient Navier-Stokes equations," Computer Methods in Applied Mechanics and Engineering, vol. 197, no. 1-4, pp. 22-35, 2007.

[12] R. Araya, G. R. Barrenechea, and F. Valentin, "A stabilized finite-element method for the Stokes problem including element and edge residuals," IMA Journal of Numerical Analysis, vol. 27, no. 1, pp. 172-197, 2007.

[13] G. R. Barrenechea and F. Valentin, "Relationship between multiscale enrichment and stabilized finite element methods for the generalized Stokes problem," Comptes Rendus Mathématique. Académie des Sciences, vol. 341, no. 10, pp. 635-640, 2005.

[14] Y. He and K. Li, "Two-level stabilized finite element methods for the steady Navier-Stokes problem," Computing, vol. 74, no. 4, pp. 337-351, 2005.

[15] L. Franca, A. Madureira, and F. Valentin, "Towards multiscale functions: enriching finite element spaces with local but not bubble-like functions," Computer Methods in Applied Mechanics and Engineering, vol. 194, pp. 2077-2094, 2005.

[16] R. Araya, G. R. Barrenechea, and F. Valentin, "Stabilized finite element methods based on multiscaled enrichment for the Stokes problem," SIAM Journal on Numerical Analysis, vol. 44, no. 1, pp. 322-348, 2006.

[17] L. P. Franca, J. V. A. Ramalho, and F. Valentin, "Multiscale finite element methods for unsteady reaction-diffusion problems," Communications in Numerical Methods in Engineering, vol. 22, no. 6, pp. 619-625, 2006.

[18] M. Crouzeix and P. A. Raviart, "Conforming and nonconforming finite element methods for the stationary Stokes equations I," RAIRO, vol. 7, pp. 33-76, 1973.

[19] L. Zhu, J. Li, and Z. Chen, "A new local stabilized nonconforming finite element method for solving stationary Navier-Stokes equations," Journal of Computational and Applied Mathematics, vol. 235, no. 8, pp. 2821-2831, 2011.

[20] R. Temam, Navier-Stokes Equation: Theory and Numerical Analysis, vol. 2, North-Holland, Amsterdam, The Netherland, 3rd edition, 1984.

[21] Z. X. Chen, Finite Element Methods and Their Applications, Springer, Heidelerg, Germany, 2005.

[22] W. Layton and L. Tobiska, "A two-level method with backtracking for the Navier-Stokes equations," SIAM Journal on Numerical Analysis, vol. 35, no. 5, pp. 2035-2054, 1998.

[23] F. Hecht, O. Pironneau, A. Le Hyaric, and K. Ohtsuka, May 2008, http://www.freefem.org/ff++ .

[24] U. Ghia, K. N. Ghia, and C. T. Shin, "High-Re solutions for incompressible flow using the NavierStokes equations and a multigrid method," Journal of Computational Physics, vol. 48, no. 3, pp. 387-411, 1982. 


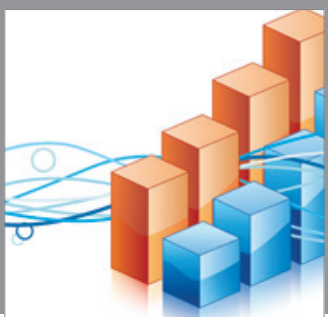

Advances in

Operations Research

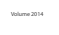

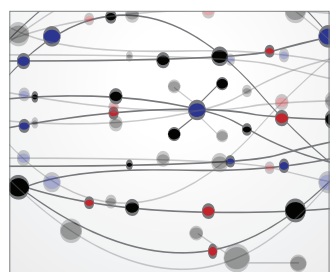

\section{The Scientific} World Journal
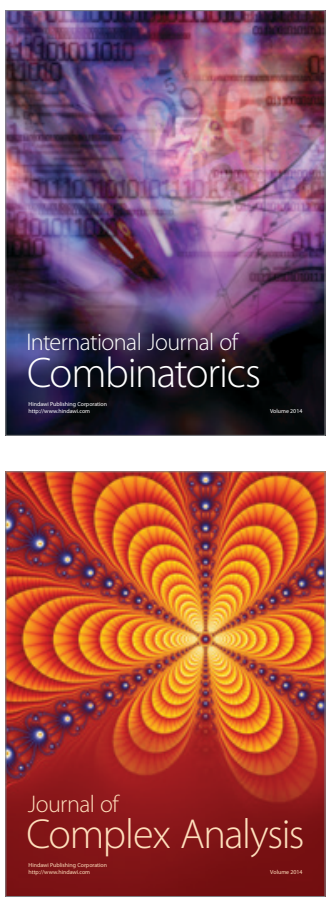

International Journal of

Mathematics and

Mathematical

Sciences
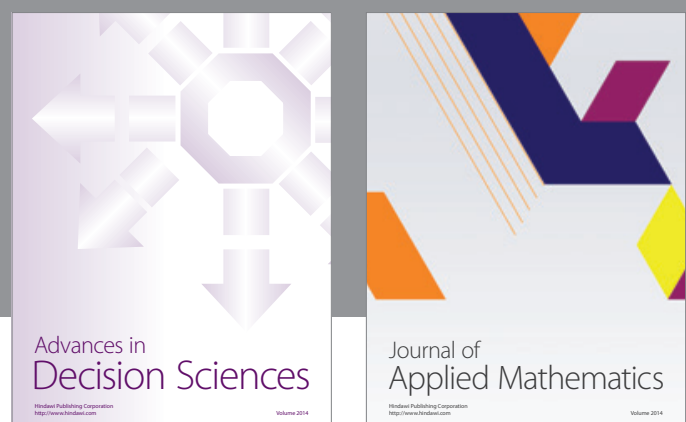

Journal of

Applied Mathematics
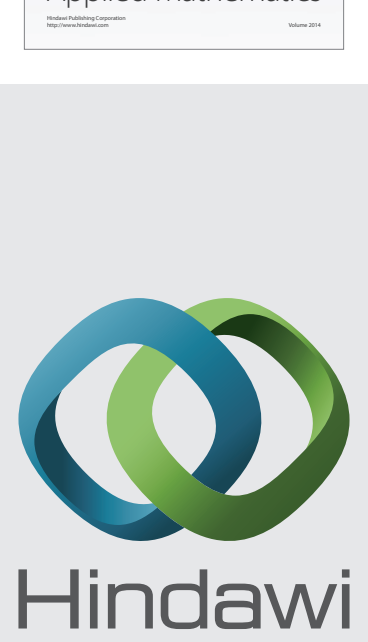

Submit your manuscripts at http://www.hindawi.com
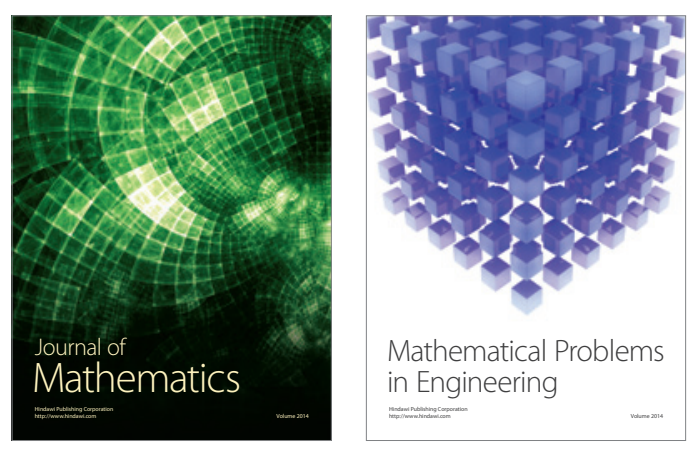

Mathematical Problems in Engineering
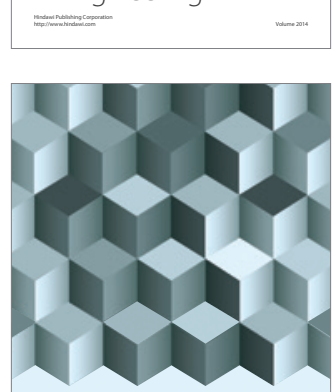

Journal of

Function Spaces
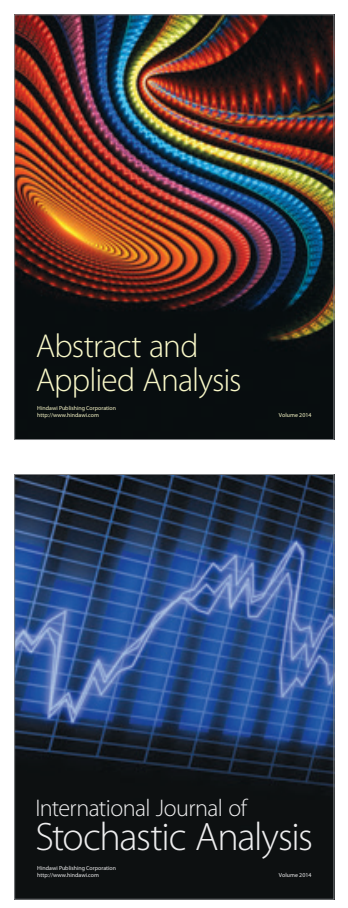

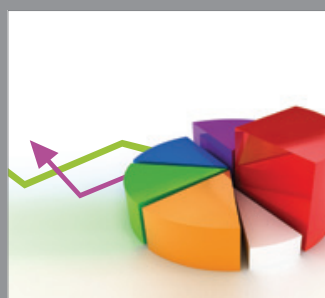

ournal of

Probability and Statistics

Promensencen
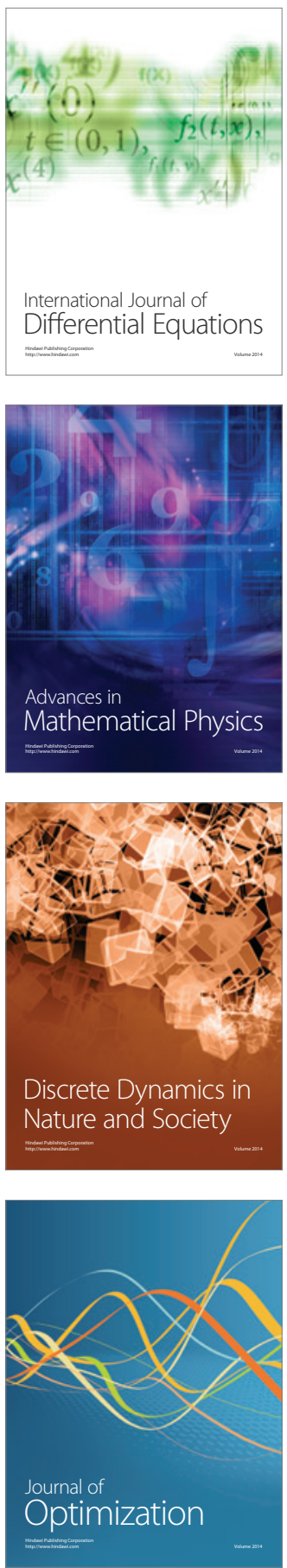